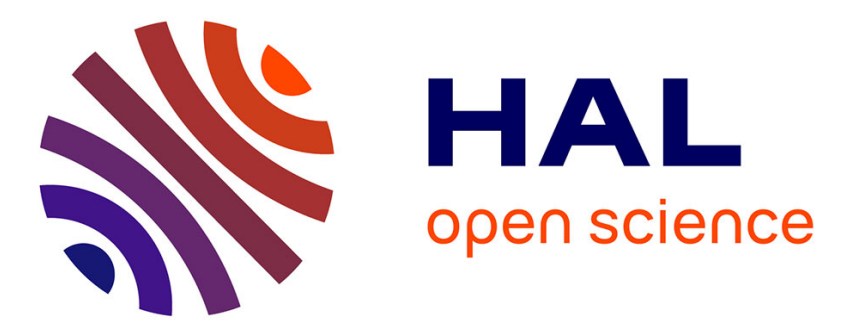

\title{
Coupling mantle convection and tidal dissipation: applications to Enceladus and Earth-like planets
}

\author{
M. Behounkova, G. Tobie, G. Choblet, O. Cadek
}

\section{To cite this version:}

M. Behounkova, G. Tobie, G. Choblet, O. Cadek. Coupling mantle convection and tidal dissipation: applications to Enceladus and Earth-like planets. Journal of Geophysical Research, 2010, 115, pp.E09011. 10.1029/2009JE003564 . hal-00558297

\section{HAL Id: hal-00558297 \\ https://hal.science/hal-00558297}

Submitted on 9 Nov 2021

HAL is a multi-disciplinary open access archive for the deposit and dissemination of scientific research documents, whether they are published or not. The documents may come from teaching and research institutions in France or abroad, or from public or private research centers.
L'archive ouverte pluridisciplinaire HAL, est destinée au dépôt et à la diffusion de documents scientifiques de niveau recherche, publiés ou non, émanant des établissements d'enseignement et de recherche français ou étrangers, des laboratoires publics ou privés. 


\title{
Coupling mantle convection and tidal dissipation: Applications to Enceladus and Earth-like planets
}

\author{
Marie Běhounková, ${ }^{1}$ Gabriel Tobie, ${ }^{2}$ Gaël Choblet, ${ }^{2}$ and Ondřej Čadek ${ }^{1}$ \\ Received 22 December 2009; revised 19 April 2010; accepted 23 April 2010; published 30 September 2010.
}

[1] Anelastic dissipation of tidal forces likely contributes to the thermal budget of several satellites of giant planets and Earth-like planets closely orbiting other stars. In order to address how tidal heating influences the thermal evolution of such bodies, we describe here a new numerical tool that solves simultaneously mantle convection and tidal dissipation in a three-dimensional spherical geometry. Since the two processes occur at different timescales, tidal dissipation averaged over a forcing period is included as a volumetric heat source for mantle dynamics. For the long-term flow, a purely viscous material is considered, whereas a Maxwell-like formalism is employed for the tidal viscoelastic problem. Due to the strongly temperature dependent rheological properties of both mechanisms, the coupling is achieved via the temperature field. The model is applied to two examples: Enceladus and an Earth-like planet. For Enceladus, our new 3-D method shows that the tidal strain rates are strongly enhanced in hot upwellings when compared with classical methods. Moreover, the heat flux at the base of Enceladus' ice shell is strongly reduced at the poles, thus favoring the preservation of a liquid reservoir at depth. For Earth-like planets, tidal dissipation patterns are predicted for different orbital configuration. Thermal runaway is observed for orbital periods smaller than a critical value (e.g., 30 days for an eccentricity of 0.2 and $3: 2$ resonance). This is likely to promote large-scale melting of the mantle and Io-like volcanism.

Citation: Běhounková, M., G. Tobie, G. Choblet, and O. Čadek (2010), Coupling mantle convection and tidal dissipation: Applications to Enceladus and Earth-like planets, J. Geophys. Res., 115, E09011, doi:10.1029/2009JE003564.

\section{Introduction}

[2] Anelastic dissipation of tidal forces constitutes a major source of energy for the interior of several bodies in the solar system. The huge volcanism on Io [e.g., Lopes and Williams, 2005] as well as the eruptions of water vapor and ice particles on Enceladus' south pole [e.g., Porco et al., 2006] are probably the best examples of internal activity triggered by tidal dissipation. Surface heat fluxes on these two bodies are so intense that they can be easily measured from infrared remote sensing [Veeder et al., 1994; Spencer et al., 2009]. The total emitted power, $\sim 100 \mathrm{TW}$ on Io and up to $\sim 15 \mathrm{GW}$ on Enceladus, exceeds by more than one order of magnitude the power that can be provided by radiogenic decay, thus definitely demonstrating that an additional source of energy exists in their interior. Even though in a less spectacular manner, tidal dissipation is also expected to significantly contribute to the energy budget of Europa and Titan [Tobie et al., 2005b]. Tidal dissipation in the outer ice shell of Europa is able to stabilize an internal ocean at about $20-30 \mathrm{~km}$ below its cold surface [Ojakangas

\footnotetext{
${ }^{1}$ Faculty of Mathematics and Physics, Department of Geophysics, Charles University in Prague, Prague, Czech Republic.

${ }^{2}$ Laboratoire de Planétologie et Géodynamique, UMR 6112, Université de Nantes, CNRS, Nantes, France.

Copyright 2010 by the American Geophysical Union. 0148-0227/10/2009JE003564
}

and Stevenson, 1989; Hussmann et al., 2002; Tobie et al., 2003]. Within Titan, tidal dissipation has also probably limited the crystallization of its internal ocean and may have triggered late cryovolcanic activity [Tobie et al., 2005a, 2006]. Tidal dissipation is also suggested to play a key role during the early stage of the Earth-Moon system, when the Moon was much closer [e.g., Ross and Schubert, 1989], as well as on Mercury during its despinning that led to the present-day 3:2 resonant orbital configuration [e.g., Robuchon et al., 2009]. Outside our solar system, a multitude of terrestrial planets are potentially affected by tidal interactions [e.g., Mardling and Lin, 2004]. For very short-period planets, tidal heating may be large enough to completely melt their interior [Jackson et al., 2008].

[3] As the anelastic properties of planetary materials (silicate minerals, ice) [e.g., Faul and Jackson, 2005; Cole, 1995] significantly depend on temperature, a strong coupling between the production of heat by tidal anelastic friction and the heat transfer by thermal convection and conduction exists, leading in some extreme cases like on Io to a thermal runaway. Modeling this feedback effect of tidal dissipation on the thermal evolution of planets and satellites is very challenging. Since the pioneer work of Peale et al. [1979] on the tidally induced melting of Io due to tidal friction, many models have been developed to describe the role of tidal heating on the evolution of giant planets' moons. However, most of them are based on a parameter- 
ized description of heat transfer and assume a constant distribution of dissipation in the mantles [e.g., Fischer and Spohn, 1990; Moore, 2003; Hussmann and Spohn, 2004]. Only few studies have tried to address the distribution of heat in the satellite interior and its impact on the temperature distribution [e.g., Segatz et al., 1988; Tackley et al., 2001; Tobie et al., 2003; Czechowski and Leliwa-Kopystynski, 2005; Roberts and Nimmo, 2008a]. In all these studies, the effect of lateral viscosity variations on the tidal response of the satellite is neglected, whereas it is expected to play a key role on the tidal heating pattern [Tobie et al., 2008].

[4] Here we outline a new method which permits us to compute simultaneously mantle convection and tidal dissipation in a 3-D spherical geometry by coupling two preexisting numerical programs. Even though convection and tides operate on completely different timescales (from hours to months for tides and from tens of thousands to hundreds of millions years for convection), coupling between the two processes can be achieved via the temperature field which controls both convective flow and viscoelastic tidal deformation. The dissipation rate, which is computed from the three-dimensional distribution of temperature determined by the convective flow and averaged over an orbital period, is imposed as an instantaneous source of volumetric heating for thermal convection. Our 3-D approach permits us to quantify the effect of tides on the thermal evolution for any kind of planetary solid objects. Here we apply our model to two examples: Enceladus and an Earth-like planet orbiting at relatively close distance from its star, where the coupling is expected to have a strong impact on the thermal evolution.

[5] Details about our model are presented in section 2 where the governing equations, their approximations and rheologies used are detailed for both mantle convection flow and viscoelastic response to tidal forcing. Numerical methods are described in section 3. Specially, the viscous flow method [Choblet et al., 2007] using finite volume approach and "cubed sphere" discretization is summarized in 3.1. Section 3.2 details the time domain approach [Čadek, 2003; Tobie et al., 2008] used for solving the viscoelastic response. In this method, discretization based on finite difference in radial direction and spherical harmonics in lateral direction is employed. The details of spatial and time coupling are provided in sections 3.3 and 3.4. Finally, the evaluation of tidal dissipation is described in section 3.5. The use of this method is illustrated for two various bodies. First, the influence of rheology is addressed for Enceladus (section 4.1). In the case of Earth-like planets, the influence of the orbital parameters on tidal dissipation is investigated (section 4.2). Technical details about the nondimensional governing equations are in Appendix A. Periodic parts of the tidal potentials for different types of orbits are provided in Appendix B. Appendix $\mathrm{C}$ deals with the benchmarks and sensitivity tests for numerical parameters.

\section{Model}

[6] As mentioned above, the tidal dissipation rate averaged over one orbital period is taken into account as a volumetric source of energy for long-term viscous flow. Since the attenuation itself depends on the temperature, this brings the necessity to solve both viscoelastic response and long-term flow simultaneously. Additionally, we concen- trate on bodies where tidal dissipation in the solid part significantly influences the thermal evolution. Typically, a silicate mantle or an icy layer (or even several icy layers in some icy satellites) is considered. We thus assume that the tidal dissipation due to anelastic processes in the other parts of the body can be approximated via boundary conditions, or is negligible. This allows us to solve the above defined problem in a spherical shell representing the mantles of the studied bodies.

\subsection{Temperature-Dependent Rheology Relevant for Convection and Tidal Dissipation}

[7] For the viscous flow of both silicate and ice, the rheological properties have rather complex character where different mechanisms (typically diffusion creep, dislocation creep, basal slip or grain boundary sliding) may prevail depending on temperature, pressure, stress, grain size, water content, porosity etc. The viscosity $\eta$ is usually described by the Arrhenius law

$$
\eta=A^{-1 / n} d^{m} \dot{\epsilon}^{(n-1) / n} \exp \left(\frac{Q^{*}}{n R T}\right),
$$

where $R$ is the gas constant. Temperature $T$, grain size $d$ and the second invariant of strain rate tensor $\dot{\epsilon}$ describe the material state. Preexponential parameter $A$, stress exponent $n$, grain size exponent $m$ and activation enthalpy $Q^{*}$ are material parameters that depend on the deformation mechanism. The values of these parameters are widely investigated for both silicates [e.g., Ashby and Verrall, 1978; Karato and Wu, 1993; Hirth and Kohlstedt, 2003; Korenaga and Karato, 2008] and icy materials [e.g., Budd and Jacka, 1989; Goldsby and Kohlstedt, 2001; Durham and Stern, 2001] for different creep regimes. As it is uncertain which creep regime prevails for silicate or ice at planetary interior conditions, we assume for simplicity a Newtonian rheology, where $n=1$ and $m=0$ in equation (1). Following the classical approach in thermal convection modeling, we employ the Frank-Kamenetskii approximation which is formulated as

$$
\eta(T)=\eta_{b} \exp \left(-a_{\text {vis }} \frac{T-T_{b}}{\Delta T}\right) .
$$

$\eta_{b}$ and $T_{b}$ are the viscosity and temperature at the base of the mantle, and $a_{\mathrm{vis}}$ corresponds to $\frac{Q^{*} \Delta T}{R T_{b}^{2}}$. For further discussion concerning the Frank-Kamenetskii approximation see, e.g., Reese et al. [1999].

[8] Due to the anelastic properties of planetary materials (ice or silicate), part of the mechanical energy associated with tidal deformation is dissipated into heat. The global anelastic response of planetary interiors to tidal forcing can be inferred from spacecraft tracking and/or altimetry [e.g., Ray et al., 2001; Yoder et al., 2003], or indirectly from secular changes of a satellite's mean motion [e.g., Lainey et al., 2009]. However, these integrate the effect of the different layers composing the body interior. The anelastic properties can also be measured experimentally for a variety of rock and ice samples. Laboratory measurements which are usually performed at frequencies higher than typical tidal frequency [e.g., Cole, 1995; Faul and Jackson, 2005] indicate that, similarly to viscous creep, the anelastic response of ice 
or rock samples depends on a variety of parameters including temperature, stress, grain size, dislocation density, water content, salinity, porosity etc. and requires the use of rather complex rheological law. Alternatively, the anelastic response can be approximated using linear viscoelastic models, such as Maxwell or Burgers rheology, that can be calibrated from existing laboratory and field data. Even though the viscoelastic linear formalism does not describe the entire complexity of anelastic response, it has the advantage to rely a limited number of parameters, whose values can be systematically explored. Here, we use the incompressible Maxwell rheology which is described by two parameters, $\eta$ the long-term viscosity and $\mu$ the elastic shear modulus. This rheology correctly describes the dissipative processes for forcing periods around or above the Maxwell time $\tau_{\mathrm{M}}=\eta / \mu$. However, it usually underestimates the dissipation rate for forcing periods much shorter than the Maxwell time [e.g., Sotin et al., 2009]. The dissipation function $Q^{-1}$ describing the energy loss during one cycle for processes with excitation angular frequency $\omega_{\mathrm{e}}$ derived for the Maxwell rheology is expressed as

$$
Q_{\text {Maxwell }}^{-1}=\left(\left(\frac{\eta \omega_{\mathrm{e}}}{\mu}\right)^{2}+1\right)^{-1 / 2} .
$$

As an example, in the case of the Earth's mantle, for a typical long-term viscosity of $10^{21} \mathrm{~Pa} \cdot \mathrm{s}$ and a shear modulus of $100 \mathrm{GPa}$ corresponding to a Maxwell time of $\sim 300$ years, the Maxwell rheology predicts a dissipation function equal to $1 / 1450000$ for semidiurnal tides. This is 5000 times smaller than the value obtained by TOPEX/ POSEIDON data [Ray et al., 2001]. However, the observed value can be reproduced with the Maxwell formalism if an effective viscosity $\hat{\eta}$, different from the inferred long-term viscosity associated with convective flow, is used:

$$
\hat{\eta}=\sqrt{Q^{2}-1} \times \frac{\hat{\mu}}{\omega_{\mathrm{e}}} .
$$

Note that the use of the effective viscosity has to be seen here as a purely mathematical parameterization of dissipation due to periodic forcing in order to reproduce the dissipation function at a given tidal frequency. In the case of the solid Earth, an effective viscosity of $\sim 2 \cdot 10^{17} \mathrm{~Pa} \cdot \mathrm{s}$ is thus required to explain the observed semidiurnal tidal dissipation. The Earth's dissipation function is also known to vary as a function of frequency [Benjamin et al., 2006] in a way which is not consistent with the frequency dependence of the Maxwell formalism. This difficulty can be overcome by defining an effective viscosity at each frequency. This is the approximation we employ here.

[9] Moreover, anelastic dissipation in water ice and rocks usually results for the combination of several distinct processes (displacement of punctual defects, dislocation glide, grain boundary sliding etc.) that are activated at different temperatures [Tatibouet et al., 1987; Cole, 1995; Faul and Jackson, 2005]. The Maxwell formalism with its single viscosity parameter can in theory describe only one dissipation process. A way of mimicking a temperature-dependent dissipation function resulting from the combination of several distinct dissipation processes activated at different tempera- tures is to consider an apparent activation energy. The combination of several dissipative processes leads to a relatively weak temperature dependence, so that the apparent activation energy is smaller than the individual activation energy associated with each attenuation process. By analogy with the mathematical approximated law used for the convective viscosity $\eta$, we consider here a temperature dependence of the effective viscosity $\hat{\eta}$ described by a single parameter $\hat{a}_{\mathrm{vis}}$ :

$$
\hat{\eta}(T)=\hat{\eta}_{b} \exp \left(-\hat{a}_{\mathrm{vis}} \frac{T-T_{b}}{\Delta T}\right) .
$$

The reference viscosity $\hat{\eta}_{b}$ is equal to the reference viscosity $\eta_{b}$ used for the convective viscosity for forcing periods close to or higher than the Maxwell time, but is usually smaller for shorter forcing period. The $\hat{a}_{\text {vis }}$ parameter is either equal to or smaller than the $a_{\mathrm{vis}}$ parameter. In the present study, we have tested different values for these two parameters.

[10] For sake of simplicity, and also in order to focus on the thermomechanical coupling between the two processes, we have chosen to use a rather simple viscosity law that depends only on temperature. Even though more complex viscosity properties should be employed to better describe the dynamics of the planets and moons (e.g., pressure dependence, possible radial stratification due to changes in composition and mineralogy, grain size, nonlinear rheology), the use of a simplified rheological description including only the temperature dependence is relevant since both viscous and anelastic deformations are primarily controlled by temperature. Note, however, that all the new numerical developments we introduce in the present study could be extended to more complex rheologies.

\subsection{Governing Equations for Mantle Convection}

[11] For mantle convection, we consider the classical Boussinesq approximation for an incompressible viscous fluid with an infinite Prandtl number. For icy moons and terrestrial bodies, the incompressible continuity equation is approximately valid and the effect of compressibility is not dramatic [Schubert et al., 2001]. The self-gravitation, which needs to be included in the viscoelastic tidal deformation (see section 2.3), is not necessary here, since its effect on the convective flow and temperature is null in the Boussinesq approximation and for free-slip or no-slip boundary conditions [Zhong et al., 2008].

[12] In this approximation, the viscous flow in the spherical shell is governed by conservation laws for mass, momentum and energy in the form

$$
\begin{gathered}
0=\nabla \cdot \boldsymbol{v}, \\
0=-\nabla p+\nabla \cdot\left(\eta(T)\left(\nabla \boldsymbol{v}+\nabla^{\mathrm{T}} \boldsymbol{v}\right)\right)-\rho_{0} \alpha\left(T-T_{\mathrm{ref}}\right) g_{0} \boldsymbol{e}_{r}, \\
\rho_{0} c_{p} \frac{\partial T}{\partial t}=-\rho_{0} c_{p} \boldsymbol{v} \cdot \nabla T+k \nabla^{2} T+h_{i}+h_{\text {tide }},
\end{gathered}
$$

where ${ }^{\mathrm{T}}$ stands for transposition. The symbols used for the model parameters and variables are listed in Table 1. Usually, the classical scaling into nondimensional variables is considered (see Table 1 where the primed variables are dimen- 
Table 1. List of Symbols and Variables ${ }^{\mathrm{a}}$

\begin{tabular}{|c|c|}
\hline Variable/Parameter & Definition \\
\hline$d=r_{t}-r_{b}$ & shell thickness \\
\hline$r_{t}$ & outer radius of the shell \\
\hline$r_{b}$ & inner radius of the shell \\
\hline$r=d r^{\prime}$ & radius \\
\hline $\mathrm{f}=\frac{r_{b}}{r}$ & geometrical factor of the shell \\
\hline & surface temperature \\
\hline$T_{b}$ & temperature on $\mathrm{CMB}$ \\
\hline$\Delta T$ & temperature contrast across the shell \\
\hline$T=T_{b}-\Delta T \theta^{\prime}$ & temperature \\
\hline \multicolumn{2}{|c|}{ Viscous Flow } \\
\hline$\rho_{0}$ & reference density \\
\hline$g_{0}$ & reference gravity acceleration \\
\hline$\kappa=\underline{k}$ & thermal diffusivity \\
\hline$\rho_{0} c_{p}$ & \\
\hline$k$ & thermal conductivity \\
\hline$c_{p}$ & heat capacity \\
\hline$\alpha$ & thermal expansivity \\
\hline$\eta_{b}$ & reference viscosity \\
\hline $\boldsymbol{v}=\frac{\kappa}{d} \boldsymbol{v}^{\prime}$ & velocity \\
\hline$t=\frac{d^{2}}{\kappa} t^{\prime}$ & time (thermal diffusion) \\
\hline$p=\frac{\eta_{b} \kappa}{d^{2}} p^{\prime}$ & dynamical pressure \\
\hline$\eta=\eta_{b} \eta^{\prime}$ & viscosity \\
\hline$h=\frac{\Delta T k}{d^{2}} h^{\prime}$ & volumetric heating \\
\hline$R a_{b}=\frac{a \rho_{0} g_{0} \Delta T d^{3}}{\eta_{b} \kappa}$ & Rayleigh number \\
\hline \multicolumn{2}{|c|}{ Viscoelastic Response } \\
\hline$\rho_{0}$ & mantle density \\
\hline$\rho_{\text {core }}$ & core density \\
\hline$g_{b}$ & gravity acceleration on surface \\
\hline$g_{s}$ & $\begin{array}{l}\text { gravity acceleration on bottom } \\
\text { boundary }\end{array}$ \\
\hline$\hat{\eta}_{b}$ & reference effective viscosity \\
\hline$\hat{\eta}=\hat{\eta}_{b} \hat{\eta}^{\prime}$ & effective viscosity \\
\hline$\hat{\mu}=d \rho_{0} g_{0} \hat{\mu}^{\prime}$ & effective shear modulus \\
\hline $\boldsymbol{u}=d \boldsymbol{u}^{\prime}$ & displacement \\
\hline$\tau=\frac{\hat{\eta}_{b}}{d \rho_{0} g_{0}} \tau^{\prime}=\tau_{0} \tau^{\prime}$ & viscous time \\
\hline$\pi=d \rho_{0} g_{0} \pi^{\prime}$ & $\begin{array}{l}\text { isotropic part of the incremental } \\
\text { stress tensor }\end{array}$ \\
\hline $\mathbf{D}=d \rho_{0} g_{0} \mathbf{D}^{\prime}$ & $\begin{array}{l}\text { deviatoric part of the incremental } \\
\text { stress tensor }\end{array}$ \\
\hline $\boldsymbol{f}=\rho_{0} g_{0} \boldsymbol{f}^{\prime}$ & body force \\
\hline
\end{tabular}

${ }^{\mathrm{a}}$ Variables with prime denote dimensionless variables.

sionless variables and Appendix A) and the vigor of the system is characterized by dimensionless Rayleigh number $R a$. As mentioned above, the rheological relationship for viscosity is supposed to be Newtonian and it is described using the dimensionless parameter $a_{\text {vis }}$ (see equation (2)).

[13] Kinematic (no-slip) or dynamic (free-slip) mechanical boundary conditions can be considered depending on the nature of the boundaries. Typically, no-slip conditions are prescribed for solid-solid boundary and free-slip boundary conditions for solid-liquid boundary. For the thermal equation, either temperature or heat flux on the boundaries is prescribed. Here, the following boundary conditions in temperature are considered $T\left(r=r_{t}\right)=T_{s}=T_{b}-\Delta T$ on the surface and $T\left(r=r_{b}\right)=T_{b}$ on the bottom boundary.

[14] In the framework of the classical Boussinesq approximation, the energy budget (equation (8)) is determined by in/out heat flux through boundaries and the volumetric heat sources $h_{i}$ and $h_{\text {tide }}$. The term $h_{i}$ includes various sources of internal energy, usually the radiogenic heating and, in the case of steady state experiments describing instantaneous heat transfer in a transient framework, secular cooling [see, e.g., Choblet and Sotin, 2000]. Additionally to this traditional term, we consider the heterogeneous energy source $h_{\text {tide }}$ induced by tidal dissipation as described in the following section.

\subsection{Viscoelastic Model for Tidal Dissipation}

[15] For sake of simplicity and in order to keep a minimal number of rheological parameters, an incompressible Maxwell model is used to describe the dissipation associated with solid tides. As illustrated by Tobie et al. [2005b], the compressibility has a limited effect on the tidal deformation and on the distribution of tidal heating, and therefore can be neglected at a first order approximation.

[16] Assuming small deformations, the governing equations for viscoelastic deformations in the Maxwell-like formalism describing conservation of mass and momentum (neglecting inertia) are

$$
\begin{gathered}
0=\nabla \cdot \boldsymbol{u} \\
0=-\nabla \pi+\nabla \cdot \mathbf{D}+\boldsymbol{f} \\
-\frac{\hat{\mu}}{\hat{\eta}} \mathbf{D}=\frac{\partial \mathbf{D}}{\partial \tau}-\frac{\partial}{\partial \tau}\left[\hat{\mu}\left(\nabla \boldsymbol{u}+\nabla^{\mathrm{T}} \boldsymbol{u}\right)\right]
\end{gathered}
$$

where $\boldsymbol{u}$ is the displacement vector, $\pi$ and $\mathbf{D}$ represents the isotropic and the deviatoric part of the incremental stress tensor, $\boldsymbol{f}$ is the body force (see Table 1). The nondimensional formulation of the viscoelastic problem is shown in Appendix A. Note that the timescales of the viscoelastic deformation and the viscous flow differ significantly. We therefore use different symbols $(t$ and $\tau$ ) for viscous flow and viscoelastic response to distinguish between them.

[17] In order to represents an incompressible Maxwell body, we use an effective viscosity $\hat{\eta}$ and an effective shear modulus $\hat{\mu}$ (see section 2.1). The temperature dependence in form equation (5) for the effective viscosity is considered. In the case of the effective shear modulus, the rheological properties of the body are simplified: either a constant $(\hat{\mu}=$ $\left.\hat{\mu}_{0}\right)$ or a depth-dependent $(\hat{\mu}=\hat{\mu}(r))$ shear modulus is taken into account.

[18] The body force $\boldsymbol{f}$ consists of two contributions:

$$
\boldsymbol{f}=\rho_{0}(\nabla \Theta+\nabla V),
$$

where $\Theta$ is the periodic part of the external tidal potential and $V$ represents the perturbations of the gravitational potential induced by boundary deflections. Formulas for the external tidal potential depending on radius $r$, colatitude $\vartheta$, longitude $\varphi$, mean anomaly, eccentricity can be derived using, e.g., Kaula [1964]. Additionally, tidal potential varies also for different types of orbit, i.e on spin-orbit period ratio (SOPR). The derived tidal potentials for the general case, 1:1 (synchronous orbit) and 3:2 resonance are shown in Appendix B up to fourth order in eccentricity. In general, the behavior of the periodic part of the tidal potential reflect the 
spin and orbital angular frequencies of a body and their linear combinations. Specifically, in the case of 1:1 and 3:2 SOPR, the potential depends on orbital period $T_{\mathrm{O}}$ and $T_{\mathrm{O}} / 2$. The range of the periods is thus strongly limited and the assumption considered for computation of effective viscosity $\hat{\eta}$ (equation (4)) is approximately valid. On the other hand, the use of this approximation may be problematic if spin and orbit periods differ significantly and if large eccentricity is considered (see formula for the general potential in Appendix B).

[19] The influence of the potential $V$ due to the induced deformation may be substantial, especially for terrestrial bodies. Contrary to a viscous flow, the effect of the additional potential for the viscoelastic response has to be included explicitly. Here, boundary conditions are in the form of equations (13) and (14), where dynamic pressure is coupled with deformations and stresses. The viscoelastic deformations are hence also influenced by the additional potential caused by the boundary deflections. According to our calculations, neglecting the potential associated with boundary deformations may produce an error of tens of percents in the resulting dissipation energy for an Earth-like body.

[20] The boundary conditions are linearized and prescribed on the undeformed boundaries. On the surface, the condition corresponds to the force equilibrium:

$$
(-\pi \mathbf{I}+\mathbf{D}) \cdot \boldsymbol{e}_{r}+u_{r} \rho_{0} g_{s} \boldsymbol{e}_{r}=\mathbf{0},
$$

where $\mathbf{I}$ is the identity matrix, $g_{s}$ is the mean gravity acceleration on the surface and $u_{r}$ denotes the radial component of the displacement. Similar boundary conditions are prescribed also on the boundary for solid-liquid interface:

$$
(-\pi \mathbf{I}+\mathbf{D}) \cdot \boldsymbol{e}_{r}-u_{r}\left(\rho_{\text {core }}-\rho_{0}\right) g_{b} \boldsymbol{e}_{r}=-\rho_{\text {core }}(\Theta+V) \boldsymbol{e}_{r},
$$

where $g_{b}$ is the mean gravity acceleration on the boundary. The additional term on the right-hand side corresponds to pressure within the liquid core or a subsurface ocean caused by the presence of potentials $\Theta$ and $V$. In the case of solidsolid interface (assuming negligible deformation within a core), a no-slip condition is prescribed:

$$
\boldsymbol{u}=\mathbf{0} .
$$

In some specific cases, as suggested, e.g., for Enceladus [Tobie et al., 2008], a combination of both conditions may be prescribed on the bottom boundary.

[21] The dissipation rate $h_{\text {tide }}$ at a given point is computed as a time average over one period. In the above described body, it is computed as follows [see, e.g., Hanyk et al., 2005]

$$
h_{\text {tide }}=\frac{1}{T} \int_{\tau}^{\tau+T} \frac{\mathbf{D}(\sigma): \mathbf{D}(\sigma)}{2 \hat{\eta}} \mathrm{d} \sigma
$$

where $\tau$ is an arbitrary time, $T$ is the period of the tides, D:D is the double scalar product corresponding to the square of the second invariant of the deviatoric stress tensor. The globally averaged dissipation production is then assessed by integrating $h_{\text {tide }}$ over the volume of the spherical shell $V$

$$
P_{\text {glob }}=\int_{V} h_{\text {tide }} \mathrm{d} V
$$

\section{Numerical Methods}

\subsection{Viscous Dynamics}

[22] The numerical solution of equations (6)-(8) for rheological properties equation (2) is described in details by Choblet [2005] and Choblet et al. [2007]. In this method, the composite mesh based on the "cubed sphere" transform [Ronchi et al., 1996] is employed. This parametrization divides the sphere into six identical blocks and allows to avoid singularities at the poles. In addition, it leads to an almost uniform grid coverage of the entire sphere. Moreover, an efficient block-oriented parallelization using $6 \times 2^{N}, N=0$, $1,2, \ldots$, processors is possible.

[23] The numerical solution of thermal convection is calculated using a finite volume method with the discretization described above. The Stokes problem (equations (6)-(7)) is solved using the FAS-multigrid method with V cycles, a Gauss-Seidel smoother and linear transfer operators, except for the prolongation of pressure where the method described by Tackley [2008] is used, favoring a better treatment of strong viscosity gradients. The treatment of the energy conservation, equation (8), is based on a Godunov-like high resolution method for the advection terms. The above described approach leads to a robust numerical tool for large viscosity contrasts.

\subsection{Viscoelastic Response}

[24] For the calculation of body tide dissipation (equation 16), the Maxwell viscoelastic response (equations (9)-(11)) to the external potential is outlined by Tobie et al. [2008] and a method similar to Čadek [1989] and Čadek et al. [1992] is used.

[25] This approach is based on integration of equations (9)-(11) directly in the time domain and allows to handle both radial and lateral variations of the effective viscosity $\hat{\eta}$. Assuming an initial state with zero deformation, the time integration of equation (11) leads to

$$
\mathbf{D}(\tau)-\hat{\mu}\left(\nabla \boldsymbol{u}(\tau)+\nabla^{\mathrm{T}} \boldsymbol{u}(\tau)\right)=-\hat{\mu} \int_{0}^{\tau} \frac{1}{\hat{\eta}} \mathbf{D} \mathrm{d} \sigma=\mathbf{M} .
$$

Equations (9)-(10) and (18) are solved implicitly in time for stress tensor $-\pi \mathbf{I}+\mathbf{D}$ and displacement field $\boldsymbol{u}$, i.e. variables $\pi_{i}, \mathbf{D}_{i}, \boldsymbol{u}_{i}$ are found in the $i$ th time step. For the memory term $\mathbf{M}$ and potential $V$, we use the explicit approach. $\mathbf{M}_{i-1}$ and $V_{i-1}$ thus form the right-hand side of the equations solved in the $i$ th time step [see Tobie et al., 2008].

[26] For spatial discretization in the lateral direction, a method employing spectral decomposition into spherical harmonics is used [see, e.g., Jones, 1985; Varshalovich et al., 1989]. As the shear modulus is assumed to be only depthdependent and the memory term $\mathbf{M}$ is included explicitly, the system of equations (9)-(10) and (18) can be classified according to degree $j$ and order $m$ and splits into sets of ordinary differential equations in $r$ for spheroidal and 
toroidal parts. Moreover, the solutions can be computed for a given $j$ only and then applied to $m=0, \ldots, j$ right-hand sides. Owing to the separation, the solution is not too time demanding for intermediate wavelengths (up to 100 ) especially when compared to the evaluation of the memory term M. In the radial direction, a staggered finite difference method is used for solving ordinary partial differential equations. The radial resolution of the mantle convection solver is considered here.

[27] Moreover, the use of spherical harmonical expansion in lateral directions is convenient for computation of additional potential $V$ induced by deformations of boundaries (see equations (12) and (14)). Here, we approximate additional mass anomalies caused by the deflections of boundaries by surface densities $\sigma$ located either on top or bottom boundary, i.e. $\sigma=\Delta \rho u_{r}$, where $\Delta \rho$ is density contrast across the boundary and $u_{r}$ is radial displacement of this boundary. Then we follow classical multipole expansion using the addition theorem for spherical harmonics in order to express analytically the potential $V$ and its gradient $\nabla V$.

[28] Considering lateral variations of viscosity $\hat{\eta}$ (hence fluidity $1 / \hat{\eta}$ ) and the stress tensor $\mathbf{D}$, the computation of the quantity $\mathbf{D} / \hat{\eta}$, necessary to evaluate the memory term, is extremely time consuming. Furthermore, this term has to be evaluated at each viscoelastic time step (VETS) and at each discrete layer. This term, i.e. multiplication of two series in spherical harmonic formalism, may be evaluated using an analytic formula [see Jones, 1985]. This approach is efficient for a low cut-off degree of expansion or for an axisymmetric case. Nevertheless, for higher degrees and a fully 3-D approach, this method becomes inefficient mostly due to calculation of Clebsch-Gordan coefficients [see, e.g., Varshalovich et al., 1989] which is a nontrivial and timedemanding operation causing large errors.

[29] Here we have adopted a technique based on Legendre and Fourier transforms developed for scalar-scalar multiplication of fields represented in the spherical harmonics basis [Martinec, 1989]. In our case, the tensor-scalar multiplication has tobe evaluated. In the first step, spherical harmonic expansion of five independent components in spherical coordinates are thus computed from the tensor $\mathbf{D}$ represented on the spherical harmonic tensor basis. For each of these components, the harmonic synthesis (i.e. the double integration) is computed in each VETS at grid nodes in spatial domain. On this grid, the product between each component and the viscosity is performed and then the harmonic analysis is applied to the resulting products. Additionally, this procedure is performed on a special grid corresponding to the $L$ roots of Legendre polynomial, cf. Gauss-Legendre quadrature formula [e.g., Press et al., 1992], in latitudinal direction. This approach enables the integration of all polynomials up to degree $2 L-1$ exactly. The number of grid points in latitudinal direction should be chosen $L>\frac{3}{2} j_{\max }$ if the cut-off degree is $j_{\max }$. In longitudinal direction, Fast Fourier Transform [see, e.g., Press et al., 1992] is performed. The number of grid points $K$ has to be $K>3 j_{\max }$ with $K$ a power of 2 . After this procedure, the term $\mathbf{D} / \hat{\eta}$ in spherical harmonic basis is recomposed from its spherical coordinates components. Moreover, the layerbased parallelization can be easily performed using the discrete layering of this problem. Owing to the interconnection between the finite-volume method for viscous convection and the combined spectral-finite difference approach, an optimal number of processors exists.

\subsection{Coupled Spatial Discretizations}

[30] In the context of the calculation of the memory term M, an important issue is connected to the evaluation of the effective viscosity. Since it is temperature dependent the coupling between the viscous flow and the viscoelastic response is directly connected to this step. Owing to large variations of the effective viscosity $\hat{\eta}$ in lateral directions, computations of spherical harmonic coefficients of the effective viscosity $\hat{\eta}$ (or fluidity $1 / \hat{\eta}$ ) may lead to large errors. Nevertheless, the evaluation of these coefficients is not necessary if the Legendre-Fourier transform described above is employed. Since the effective viscosity is considered to be temperature-dependent, only the temperature field on the grid for Legendre-Fourier transform is needed and then the viscosity itself on this auxiliary grid can be computed using equation (5).

[31] The coupling between mantle flow and viscoelastic response is thus linked to the interpolation of temperature from the mantle convection grid using "cubed sphere" coordinates into the auxiliary grid for the Legendre-Fourier transform. Several possibilities have been tested to perform such a task. Specifically, two main approaches are: First, the spherical harmonic coefficients of temperature are computed using a direct integration procedure, i.e. the coefficients are directly evaluated using the definition of spherical harmonic expansion and the orthonormality of spherical harmonic functions. Then the temperature is evaluated on the auxiliary grid employing spherical harmonic synthesis. This method is highly accurate and can be parallelized. However, it is not possible to use the highly efficient Legendre-Fourier transform in this approach because the temperature field is originally computed and stored in the mantle-convection discrete framework. The second approach simply employs the properties of the "cubed sphere" coordinates where a spherical surface is mapped onto a rectangular grid. In a given discrete layer, a simple bilinear interpolation of temperature from mantle convection grid to auxiliary LegendreFourier grid in "cubed sphere" coordinates is evaluated. This technique is efficient and it can be easily and efficiently parallelized. Nevertheless, it produces an error if the power of the temperature field on degrees higher than cut-off degree $j_{\max }$ is still high. The first and less effective approach should be thus employed if the cut-off degree is low and the second and more effective one is used for high cut-off degree.

\subsection{Time Evolution and Time Coupling}

[32] As mentioned above, the Maxwell viscoelastic response is solved in the time domain. The time integration of equations (9)-(10) and (18) has to be performed until a relaxed solution is found. The relaxed solution is defined here as a state for which the total dissipation $P_{\text {glob }}$ does not change with time, i.e. $P_{\text {glob }}$ does not depend on choice of $\tau$ in equation (17). In practice, the change of global dissipation has to be smaller than a chosen criterion $c$ (typically $10^{-7}-10^{-8}$ )

$$
\frac{\left|P_{\text {glob }}^{\tau_{i+1}}-P_{\text {glob }}^{\tau_{i}}\right|}{P_{\text {glob }}^{\tau_{i+1}}}<c
$$


where $\tau_{i+1}=\tau_{i}+\Delta \tau$. Additionally, since the spherical harmonic coefficients of quantities $\mathbf{D} / \hat{\eta}$ and $\mathbf{D}$ are known, the evaluation of global dissipation (and hence the use of this convergence criterion) is efficient using the orthonormality of spherical harmonics.

[33] Finding a relaxed solution using the time integration is time consuming mainly for the 0 th mantle convection time step $(t=0)$ where the solution is integrated from no deformation state $(\mathbf{D} \equiv \mathbf{0}$ and $\boldsymbol{u} \equiv \mathbf{0})$ at time $\tau_{0}$. The integration of equation (11) for 0th mantle convection step (MCTS) is performed up to time $\tau_{1}$ for which the convergence criterion equation (19) is satisfied. By this procedure, the 0th MCTS relaxed solution $\left(\boldsymbol{u}\left(\tau_{1}\right)\right.$ and $\left.\mathbf{D}\left(\tau_{1}\right)\right)$ is obtained. For later MCTS, the integration is started from the previous one so that only the correction to the previous solution is found. The relaxed solution is again assessed by satisfying the global dissipation criterion.

[34] Another important question is the updating cycle of tidal heating compared to the performed mantle convection time steps. Since the mantle-convection time step is determined by the Courant stability criterion (including the effect of large amounts of volumetric heating), the maximal possible change of the temperature field is ensured. Then we have to carefully choose the number of mantle-convection time steps after which the tidal dissipation is recomputed. The influence of a given value of MCTS is discussed in section $\mathrm{C} 2$.

\subsection{Tidal Dissipation}

[35] The spatial distribution of the mean tidal dissipation is computed after the relaxed solution $\left(\tau=\tau_{i}\right)$ is reached. However, contrary to the computation of global tidal dissipation, the evaluation of spatial distribution is a rather complex operation similar to the evaluation of the memory term M. Since the temperature field, hence the effective viscosity, is close to a constant (its change is negligible on the timescale corresponding to tides), equation (16) may be rearranged into

$$
h_{\text {tide }}=\frac{1}{T} \frac{1}{2 \hat{\eta}} \int_{\tau}^{\tau+T} \mathbf{D}: \mathbf{D d} \sigma .
$$

In practice, the period average of the variable $\mathbf{D}: \mathbf{D}$ has to be calculated. First, the spherical harmonic coefficients corresponding to the Cartesian components of tensor $\mathbf{D}$ are computed. Subsequently, each independent component is evaluated on the auxiliary grid. Then the field of product D : $\mathbf{D}=\sum_{i, j} D_{i j} D_{j i}$ is calculated where $D_{i j}$ are Cartesian components of tensor $\mathbf{D}$. This routine is repeated in each VETS after reaching the relaxed solution and the average of this field is calculated as follows:

$$
\overline{\mathbf{D}: \mathbf{D}}=\frac{1}{n} \sum_{k=1}^{n} \mathbf{D}\left(\tau_{k}\right): \mathbf{D}\left(\tau_{k}\right)
$$

where $n$ is number of VETS per period and $\tau_{k}=\tau_{k-1}+\Delta \tau$. Then the spherical harmonic coefficients of $\overline{\mathbf{D}: \mathbf{D}}$ are evaluated. This procedure using auxiliary grid leads to higher time efficiency since the highly effective LegendreFourier transform can be employed. Then the spherical harmonic synthesis of this field is computed on the grid nodes connected to the mantle convection grid. In order to obtain spatial distribution of the tidal dissipation $h_{\text {tide }}$, the averaged field $\overline{\mathbf{D}: \mathbf{D}}$ is then divided by $2 \hat{\eta}=2 \hat{\eta}_{b} \exp \left(\hat{a}_{\mathrm{vis}} \frac{T-T_{b}}{\Delta T}\right)$ where temperature $T$ is the original (noninterpolated) temperature in the mantle convection grid nodes. Additionally, this procedure leads to the reduction of the error due to interpolation of the temperature field to the auxiliary grid when used cut-off degree of the spherical harmonics coefficients is underestimated.

\section{Results for Enceladus and Earth-Like Planets}

[36] In order to demonstrate the variety of possible applications and of possible tidal potentials, we show here results of the coupled problem for two different types of bodies. First, we apply our technique to Enceladus. We assume the presence of a global liquid layer at the base of the icy mantle, even though, as suggested by Tobie et al. [2008], this layer might only exist in the southern hemisphere. As a second example, we investigate tidal dissipation in solid exoplanets assuming an internal structure similar to the Earth and considering various orbital configurations.

[37] In both cases, we first compute a fully developed pattern of the mantle convection corresponding to a given Rayleigh number and boundary conditions without tidal dissipation, i.e. we reach a state where boundary layers are fully developed and the temperature profile is in a statistical steady state. Then we "turn on" the tidal dissipation and we aim at reaching a new statistical steady state, if any. Finally, we compare these temperature profiles and patterns of mantle convection with the ones prescribed as initial conditions in order to analyze the effect of tidal dissipation.

\subsection{Enceladus}

[38] The parameters used for Enceladus are listed in Table 2 and a synchronous eccentric orbit is considered (see Appendix B). In this model, we consider a mantle consisting of an ice I which is decoupled from the silicate core by a thin liquid layer. This means that at the base of the mantle, temperature corresponding to the melting temperature $T_{m} \sim 273 \mathrm{~K}$ of mantle material is imposed. Viscosity for the melting point within the ice I material is $10^{13-15} \mathrm{~Pa} \cdot \mathrm{s}$ [Deschamps and Sotin, 2001; Tobie et al., 2003; Mitri and Showman, 2008]. We use here a value of $10^{14} \mathrm{~Pa} \cdot \mathrm{s}$ for the viscosity at the melting point (i.e. at the base of the layer, $\left.\eta_{b}\right)$. The viscosity parameter $a_{\text {vis }}$ is set to 15 which corresponds to $Q^{*} \approx 45 \mathrm{~kJ} \cdot \mathrm{mol}^{-1}$, hence it is somewhat smaller than in, e.g., Goldsby and Kohlstedt [2001]. Additionally, an even lower value of $a_{\mathrm{vis}}=10$ is tested following Barr [2008], who suggested in the case of Enceladus a mobile lid convection rather than stagnant lid convection to explain the observed heat flux.

[39] For the effective viscosity, we use two values, $\hat{\eta}=$ $10^{14} \mathrm{~Pa} \cdot \mathrm{s}$ and $10^{13} \mathrm{~Pa} \cdot \mathrm{s}$, in order to study the sensitivity of the tidal dissipation on the effective viscosity. For the effective viscosity parameter $\hat{a}_{\text {vis }}$, two cases are investigated: i) $a_{\text {vis }}=\hat{a}_{\text {vis }}=15$ (or $a_{\text {vis }}=\hat{a}_{\text {vis }}=10$ ), i.e. the same temperature dependence is considered for both viscous flow and viscoelastic response, and ii) $a_{\mathrm{vis}}=15$ and $\hat{a}_{\mathrm{vis}}=10$, i.e. the case where effective viscosity $\hat{\eta}$ depends less on temperature than flow viscosity $\eta$. The latter case is considered in order to better reproduce the temperature dependence of $Q$ factor derived from laboratory measurements (see section 2.1). The 
Table 2. Physical Properties and Parameters for Enceladus and Earth-Like Exoplanet

\begin{tabular}{|c|c|c|c|}
\hline Definition & Variable/Parameter & Enceladus & Earth-Like Planet \\
\hline Density within the ice shell/mantle & $\rho_{0}$ & $1000 \mathrm{~kg} \cdot \mathrm{m}^{-3}$ & $4500 \mathrm{~kg} \cdot \mathrm{m}^{-3}$ \\
\hline Density of the internal ocean & $\rho_{L}$ & $1000 \mathrm{~kg} \cdot \mathrm{m}^{-3}$ & - \\
\hline Density of the core & $\rho_{\mathrm{C}}$ & $3000 \mathrm{~kg} \cdot \mathrm{m}^{-3}$ & $11000 \mathrm{~kg} \cdot \mathrm{m}^{-3}$ \\
\hline Density of the inner core & $\rho_{\mathrm{IC}}$ & - & $13000 \mathrm{~kg} \cdot \mathrm{m}^{-3}$ \\
\hline Reference gravity acceleration & $g_{0}$ & $0.113 \mathrm{~m} \cdot \mathrm{s}^{-2}$ & $9 \mathrm{~m} \cdot \mathrm{s}^{-2}$ \\
\hline Ice shell/mantle thickness & $d$ & $82.8 \mathrm{~km}$ & $3200 \mathrm{~km}$ \\
\hline Outer radius of the ice shell/mantle & $r_{t}$ & $252.3 \mathrm{~km}$ & $6400 \mathrm{~km}$ \\
\hline Inner radius of the ice shell/mantle & $r_{b}$ & $169.5 \mathrm{~km}$ & $3200 \mathrm{~km}$ \\
\hline Geometry factor & $f$ & 0.67 & 0.5 \\
\hline Thickness of the liquid layer between core and ice shell & $r_{\mathrm{L}}$ & $0.1 \mathrm{~km}$ & - \\
\hline Inner core radius & $r_{\mathrm{IC}}$ & - & $1250 \mathrm{~km}$ \\
\hline Temperature contrast across the shell & $\Delta T$ & $200 \mathrm{~K}$ & $2500 \mathrm{~K}$ \\
\hline Temperature at the surface & $T_{s}$ & $75 \mathrm{~K}$ & $460-735 \mathrm{~K}$ \\
\hline Thermal diffusivity & $\kappa$ & $1.2 \cdot 10^{-6} \mathrm{~m}^{2} \cdot \mathrm{s}^{-1}$ & $9.25 \cdot 10^{-7} \mathrm{~m}^{2} \cdot \mathrm{s}^{-1}$ \\
\hline Thermal conductivity & $k$ & $2.3 \mathrm{~W} \cdot \mathrm{m}^{-1} \cdot \mathrm{K}^{-1}$ & $5 \mathrm{~W} \cdot \mathrm{m}^{-1} \cdot \mathrm{K}^{-1}$ \\
\hline Thermal expansivity & $\alpha$ & $1.6 \cdot 10^{-4} \mathrm{~K}^{-1}$ & $2 \cdot 10^{-5} \mathrm{~K}^{-1}$ \\
\hline Rayleigh number at the bottom boundary & $R a_{b}$ & $1.7 \cdot 10^{7}$ & $7.2 \cdot 10^{7}$ \\
\hline Viscosity at the bottom boundary & $\eta_{b}$ & $10^{14} \mathrm{~Pa} \cdot \mathrm{s}$ & $10^{21} \mathrm{~Pa} \cdot \mathrm{s}$ \\
\hline Viscosity parameter & $a_{\mathrm{vis}}$ & $10-15$ & 15 \\
\hline Effective viscosity at the bottom boundary & $\hat{\eta}_{b}$ & $10^{13-14} \mathrm{~Pa} \cdot \mathrm{s}$ & $10^{17-19} \mathrm{~Pa} \cdot \mathrm{s}$ \\
\hline Shear modulus of the ice shell/mantle & $\mu$ & $3.3 \cdot 10^{9} \mathrm{~Pa}$ & $200 \cdot 10^{9} \mathrm{~Pa}$ \\
\hline Effective viscosity parameter & $\hat{a}_{\mathrm{vis}}$ & $10-15$ & 15 \\
\hline Spin-orbit period ratio & SOPR & $1: 1$ & $3: 2 / 1: 1 / \mathrm{LR}$ \\
\hline Eccentricity & $e$ & 0.0045 & $0-0.2$ \\
\hline Orbital period & $T_{\mathrm{O}}$ & 1.37 day & $20-80$ day \\
\hline Spin period & $T_{\mathrm{R}}$ & 1.37 day & $1-60$ day \\
\hline
\end{tabular}

considered models and their rheologies are summarized in Table 3.

[40] The boundary conditions for the viscoelastic response at the top boundary follows equation (13) and at the bottom boundary equation (14). For the viscous flow, a free-slip condition is prescribed at the bottom boundary. At the top boundary, no-slip condition is used. Constant values of temperature are prescribed on both boundaries. In order to solve the viscous flow, we use $64 \times 64 \times 64$ grid points in each identical block. For the viscoelastic response, the resolution is also 64 layers in the radial direction and the lateral resolution is characterized by cut-off degree $j_{\max }=80$. The convergence parameter for the relaxed solution is chosen to be $c=5 \cdot 10^{-7}$. The response to the tidal forcing is updated every 200 MCTS.

\subsubsection{Global Time Evolution}

[41] As already mentioned, here we focus mainly on the influence of the effective rheological properties on tidal dissipation and hence on thermal evolution. The results for the above described models are given in Figures 1 (time evolution) and 2 (statistical steady state solutions).

[42] The first three models (E1-E3) have the same rheological properties for viscous flow $\left(\eta_{b}=10^{14} \mathrm{~Pa} \cdot \mathrm{s}\right.$ and $a_{\mathrm{vis}}=$ 15). The initial thermal conditions are hence identical, the bottom boundary layer and the stagnant lid develop and the internal temperature is $T \sim 245 \mathrm{~K}$. The convection pattern is characterized by small polygonal cells where quadrilaterals are predominant.

[43] In the first model (E1), the same viscosity parameters are also considered to describe the viscoelastic response. For these parameters, the global dissipation $P_{\text {glob }}$ is equal to $0.3 \mathrm{GW}$ at the beginning of the simulation. The released energy is thus an order of magnitude lower than the observed value, i.e. up to $15 \mathrm{GW}$ [Spencer et al., 2009]. The global dissipation (Figure 1a, left) slightly increases (by $5 \%$ ) during the whole simulation. This heating has however no significant influence neither on the convection pattern nor on the internal temperature and the temperature profile (Figure 1a, right) remains almost constant.

[44] Model E2 considers the same dependence of the effective viscosity on temperature $\left(\hat{a}_{\mathrm{vis}}=15\right)$. However, the effective viscosity is reduced to $10^{13} \mathrm{~Pa} \cdot \mathrm{s}$ at the bottom boundary. The tidal dissipation increases by approximately an order of magnitude in comparison with the previous model and reaches a value of the same order as the one observed for the real Enceladus. Moreover, since the tidal dissipation increases in this case with temperature and the effective viscosity is temperature-dependent, a positive feedback between tidal dissipation and internal temperature occurs (Figure 1b, left). This effect leads to a new equilibrium. For this model, the amount of global tidal dissipation in the statistical steady state is approximately twice higher ( $\sim 6 \mathrm{GW})$ than at the beginning of the simulation. The internal temperature increases (Figure 1b, right) to $\sim 260 \mathrm{~K}$, i.e. by $15 \mathrm{~K}$. Furthermore, the temperature is slightly higher under the stagnant lid than above the lower boundary layer (i.e. a subadiabatic gradient is observed), a classical feature known to occur when significant amounts of internal heating are introduced [e.g., Parmentier et al., 1994]. This effect will also favor melting at the base of the stagnant lid [Sotin et al., 2002; Tobie et al., 2008].

[45] In model E3, a different temperature dependence of the effective viscosity is employed $\left(\hat{a}_{\mathrm{vis}}=10\right)$ for the same

Table 3. Enceladus Models: Description of Rheological Properties

\begin{tabular}{lcccc}
\hline Model & $\eta_{b}(\mathrm{~Pa} \cdot \mathrm{s})$ & $a_{\text {vis }}$ & $\hat{\eta}_{b}(\mathrm{~Pa} \cdot \mathrm{s})$ & $\hat{a}_{\text {vis }}$ \\
\hline E1 & $10^{14}$ & 15 & $10^{14}$ & 15 \\
E2 & $10^{14}$ & 15 & $10^{13}$ & 15 \\
E3 & $10^{14}$ & 15 & $10^{13}$ & 10 \\
E4 & $10^{14}$ & 10 & $10^{13}$ & 10 \\
\hline
\end{tabular}



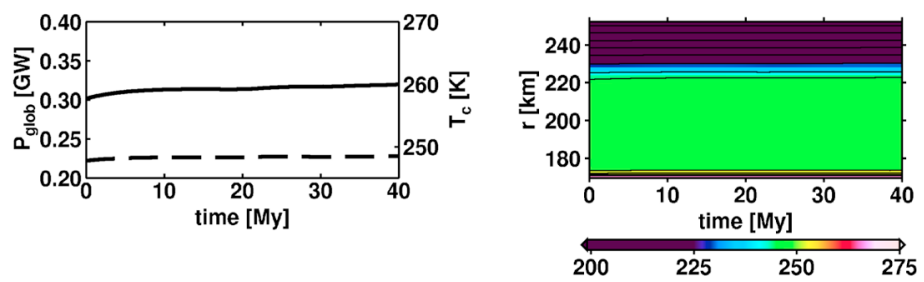

$B(E 2)$
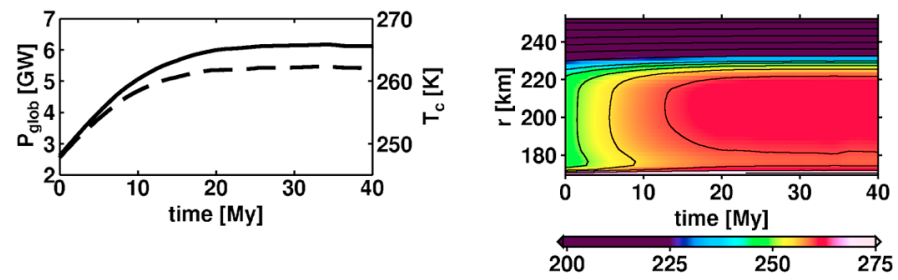

C (E3)
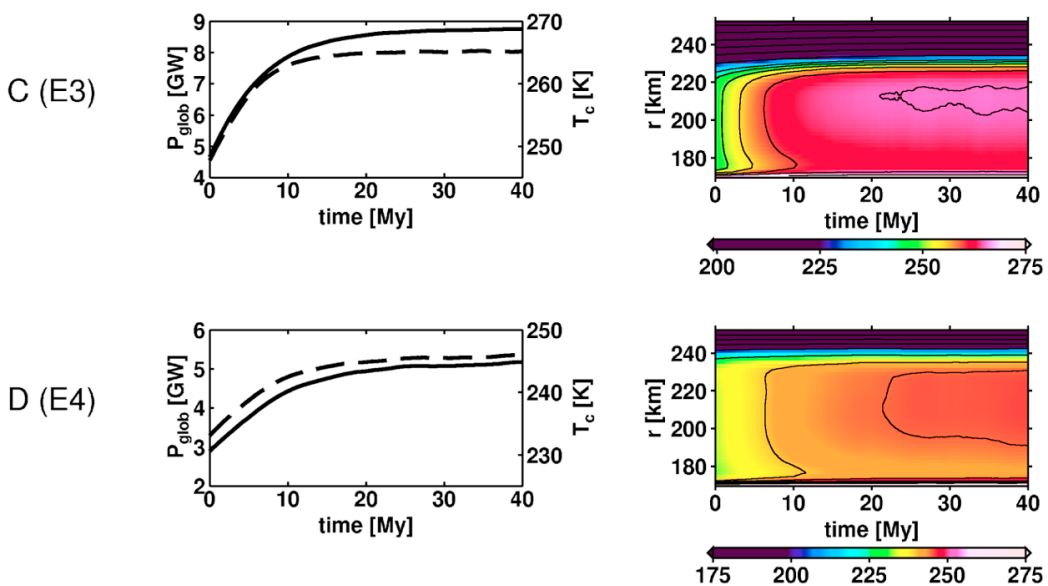

Figure 1. Enceladus model: results for models (a) E1, (b) E2, (c) E3, and (d) E4. (left) Time evolution of the global heating $P_{\text {glob }}$ (solid line) and temperature $T_{\mathrm{c}}$ (dashed line) at middle depth of the shell. (right) Time evolution of the radial temperature profile (in $\mathrm{K}$ ) with time. Contours are plotted for every $25 \mathrm{~K}$ in the lid and $5 \mathrm{~K}$ in the convective part.

effective viscosity at lower boundary $\left(\hat{\eta}_{b}=10^{13} \mathrm{~Pa} \cdot \mathrm{s}\right)$ as considered for model E2. This causes a decrease of the effective viscosity and increase in the tidal dissipation within the whole mantle in comparison with the previous case. Initially, due to the lower effective viscosity, the global tidal dissipation is higher ( $\sim 4.6 \mathrm{GW}$, Figure $1 \mathrm{c}$, left) than in model E2. Then again the positive feedback effect occurs and the dissipation increases to a statistically stable value of 8.8 GW. Owing to higher global dissipation, the internal temperature (Figure 1c, right) increases in this case by $20 \mathrm{~K}$ (to a value $\sim 265 \mathrm{~K}$ ). Additionally, a slight decrease of the thickness of the stagnant lid is observed for this model.

[46] In model E4, the flow viscosity parameter $\eta_{b}$ remains $10^{14} \mathrm{~Pa} \cdot \mathrm{s}$ but the dependence of viscosity on temperature is smaller $\left(a_{\mathrm{vis}}=10\right)$. This results in a lower internal initial temperature $(230 \mathrm{~K})$ and significantly thinner lid at the upper boundary than in the previous cases. The global dissipation at the beginning of the simulation (Figure 1d, left) is $2.9 \mathrm{GW}$, thus slightly higher than for case E2. The influence of the lower internal initial temperature is hence counter balanced by a reduction of the effective viscosity parameter $\hat{a}_{\text {vis }}$ to 10 . Nevertheless, due to the lower effect of the positive feedback caused by the lower dependence of the effective viscosity on temperature, the global dissipation for statistical steady state increases only to $5.2 \mathrm{GW}$, i.e. to a value lower than obtained in model E2. The time evolution of the temperature is shown in Figure 1d (right) (note the different color scale). Again, the temperature increases with time and the internal temperature increases to a value $\sim 245 \mathrm{~K}$ for statistical steady solution.

\subsubsection{Dissipative and Convective Patterns}

[47] The spatial distribution of temperature, tidal dissipation and heat flux for models in a statistical steady state are illustrated in Figure 2. Snapshots of temperature patterns are shown in Figure 2a for a depth just above the bottom boundary and in Figure 2c for meridian cross section at $\varphi=$ $0^{\circ}$. For model E1 (Figures 2a and 2c), the characteristics of the temperature field persist without any significant change during the whole simulation due to the low tidal dissipation rate. As the tidal dissipation rate increases (models E2-E4, Figures $2 \mathrm{a}$ and $2 \mathrm{c}$ ), the temperature patterns start to change. The spatial distribution of tidal heating breaks the relatively uniform distribution of convective planforms (Figure 2a, model E1) and favors the focusing of hot upwellings and cold downwellings in specific regions; it also affects the convective wavelength. Specifically, hot upwellings concentrate into the polar regions of the mantle, where the tidal dissipation is the highest and they elongates in north-south 

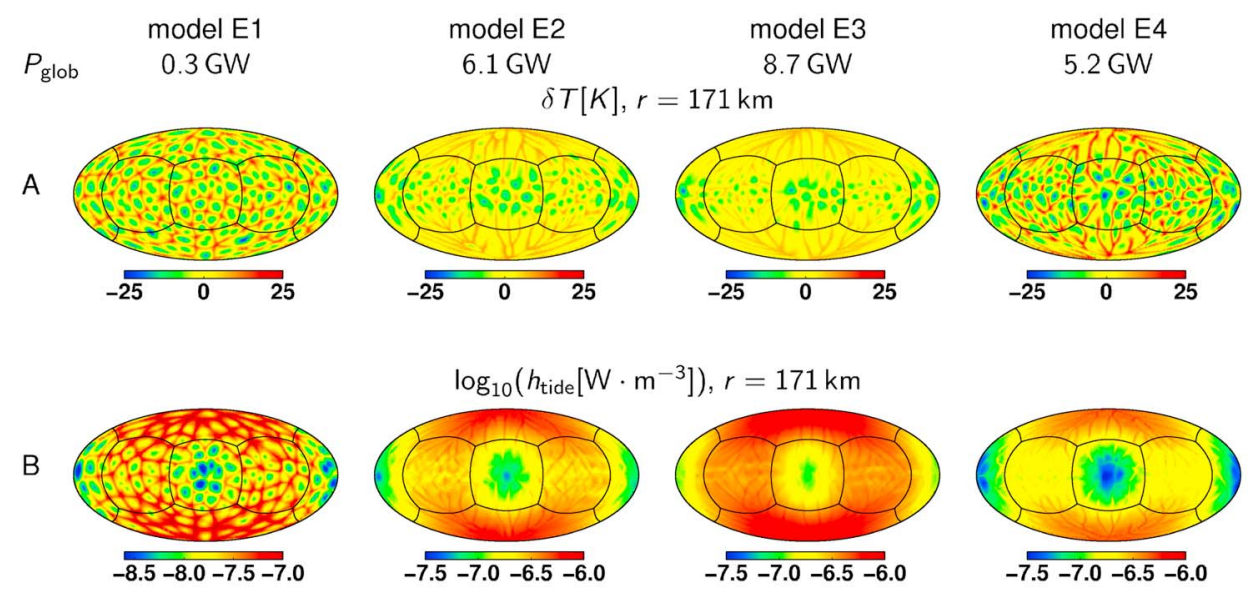

C
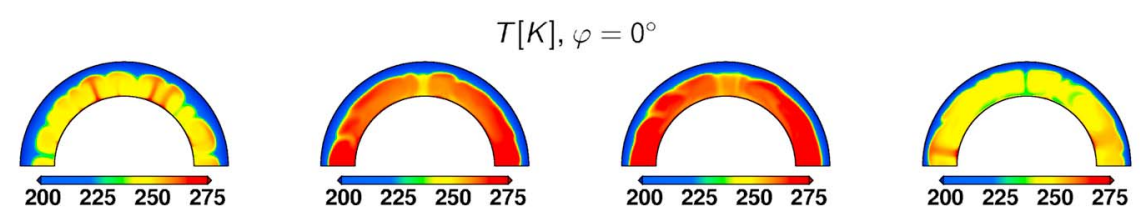

D
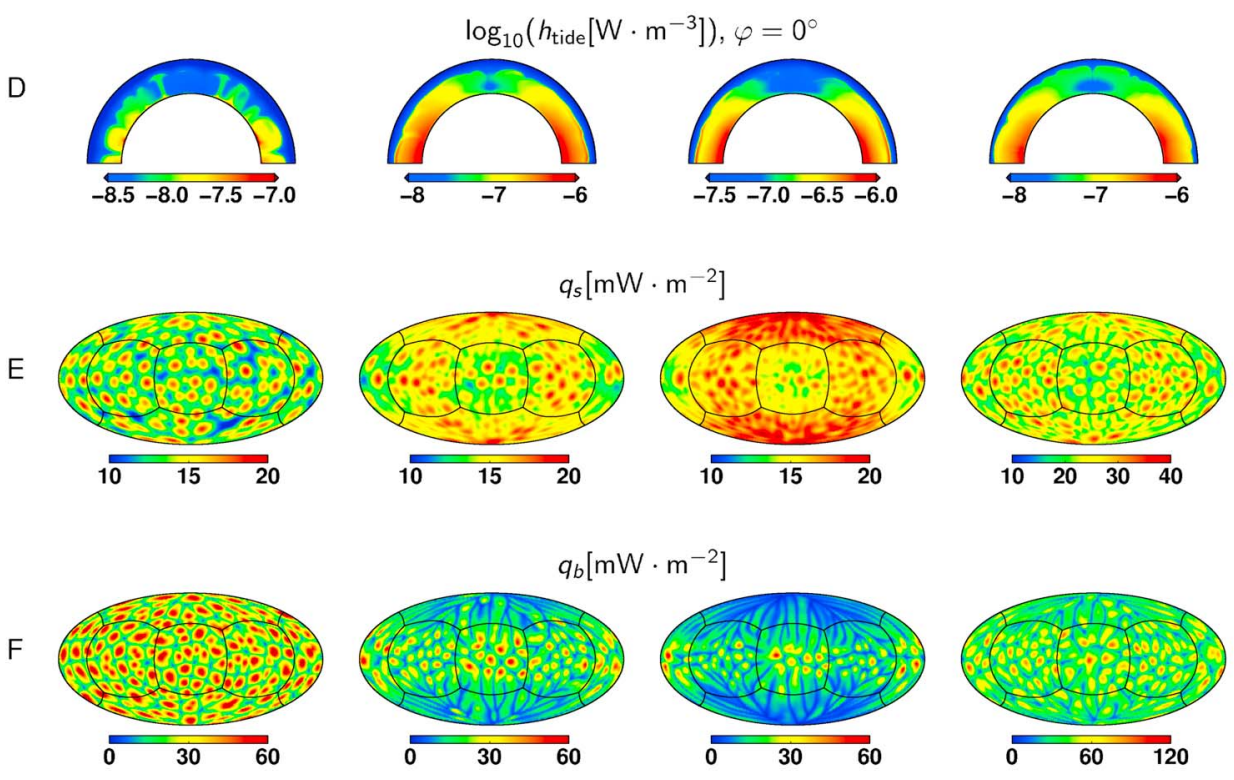

Figure 2. Enceladus model: statistical steady state solution at the time $40 \mathrm{My}$ for model E1, model E2, model E3, and model E4. (a) Temperature anomaly $\delta T$ at the depth $r=171 \mathrm{~km}$, (b) $\log 10$ of tidal dissipation at the depth $r=171 \mathrm{~km}$, (c) temperature $T$ for longitude $\varphi=0^{\circ}$, (d) $\log 10$ of tidal dissipation for longitude $\varphi=0^{\circ}$, (e) heat flux at the surface, and (f) heat flux at the bottom boundary.

direction creating sheet-like structures near the bottom boundary (Figures 3a-3c). Cold downwellings are located in regions where the dissipation rate is low. Additionally, downwellings are broader in regions where the dissipation rate is lower (they have higher characteristic wavelengths). This effect is the most pronounced in models E2 and E3 where the dissipation is highest but it is also present, to a lesser extent, in model E4.

[48] The tidal dissipation patterns near the bottom boundary (Figure 2b) are characterized by maxima at the poles and two antipodal minima on the equator. The enhance- ment toward the poles is associated with the presence of the decoupling liquid layer. The longitudinal variations are related to the 1:1 SOPR configuration. The pattern is however changing with depth. In the convecting part of the mantle, the minima remain approximately at the same positions even though their shape is changing. In the cold conductive part, the pattern is more complex although the maxima of tidal dissipation are still located at the poles. This contribution to the energy budget is however negligible since the effective viscosity is several orders of magnitude larger than in the convecting part (see Figure 2d). 
$\delta T[\mathrm{~K}]$

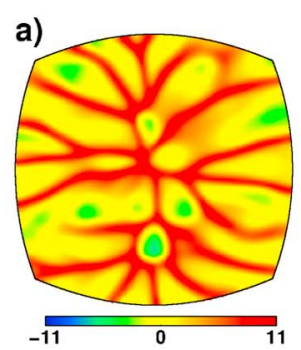

b)

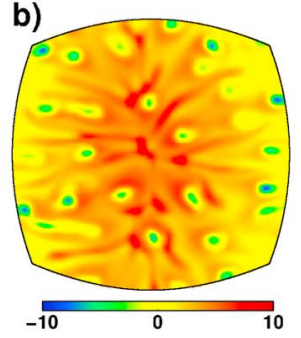

c)

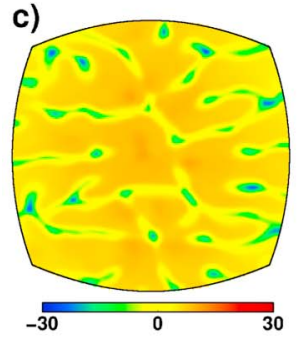

$\overline{\dot{\varepsilon} \cdot \dot{\varepsilon}} / \overline{\dot{\varepsilon}: \dot{\varepsilon}_{\text {rat }}}$

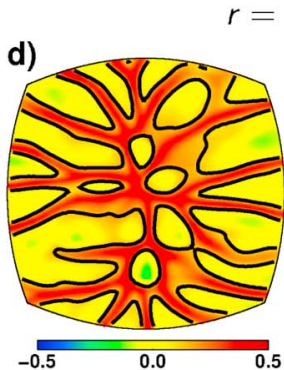

g)

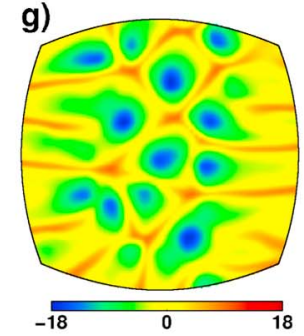

$r=195 \mathrm{~km}$

e)

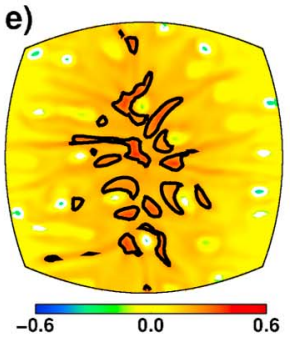

h)

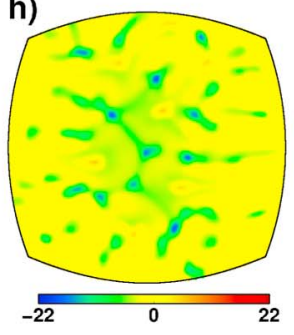

$r=220 \mathrm{~km}$

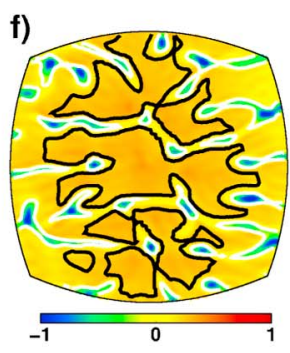

$\delta T[\mathrm{~K}]$

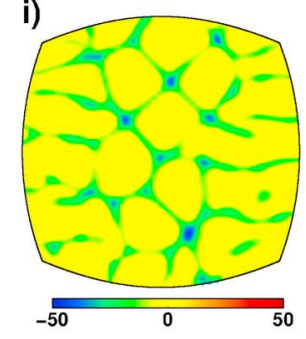

$\overline{\dot{\varepsilon}: \dot{\varepsilon}} / \overline{\dot{\varepsilon}: \dot{\varepsilon}_{\mathrm{rad}}}$

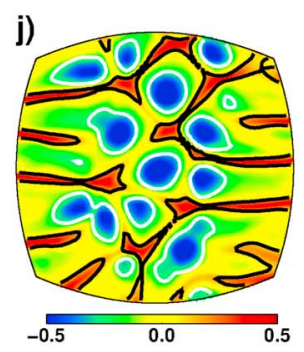

k)

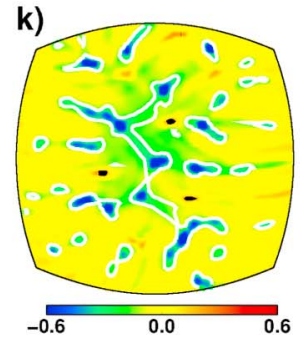

I)

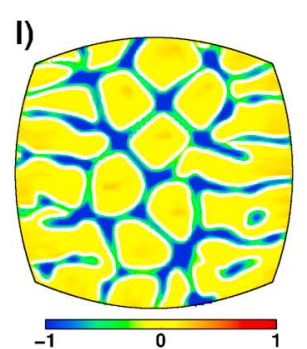

Figure 3. Enceladus model: characteristics of model $\mathrm{E} 2$ for statistical steady state $(t=40 \mathrm{My})$ : horizontal sections at three depths (a, d, g, and j) $r=171 \mathrm{~km},(\mathrm{~b}, \mathrm{e}, \mathrm{h}$, and k) $r=195 \mathrm{~km}$, and (c, f, i, and l) $r=220 \mathrm{~km}$ in the southern polar region (Figures 3a-3f, the south pole is located in the center of all these plots) and on the equator (Figures $3 \mathrm{~g}-31$, focus on a region where a minimum of tidal dissipation is located); Figures $3 \mathrm{a}-3 \mathrm{c}$ and $3 \mathrm{~g}-3 \mathrm{i}$ are temperature anomalies in $\mathrm{K}$; Figures $3 \mathrm{~d}-3 \mathrm{f}$ and $3 \mathrm{j}-31$ are $\log _{10}\left(\overline{\dot{\varepsilon}: \dot{\varepsilon}} / \dot{\dot{\varepsilon}: \dot{\varepsilon}_{\text {rad }}}\right)$; black lines denote isotherm $267 \mathrm{~K}$, and white lines denote isotherm $257 \mathrm{~K}$.

[49] Although the global pattern of tidal dissipation is primarily predetermined by the orbital parameters and the presence of the liquid layer at depth, the influence of the temperature variations is also significant: tidal dissipation is reduced considerably within cold downwellings and enhanced in hot upwellings. The low viscosity associated with hot upwellings naturally results in an increase of tidal dissipation as the dissipation rate is inversely proportional to the effective viscosity (equation (16)). Moreover, it can be seen in Figures $3 \mathrm{~d}-3 \mathrm{f}$ that the tidal strain rate is also significantly enhanced within hot upwellings. For further discussion of the influence of 3-D viscosity variations, see section 4.1.3.

[50] Besides the global dissipation, the energy budget is also characterized by the surface and bottom heat fluxes (Figures 2e and 2f). Due to the introduction of internal heat sources, the surface heat flux increases while the bottom heat flux decreases. In model E1 (Figures 2e and 2f) where no significant influence of the tidal dissipation is observed, both heat fluxes are similar to those in the initial state. In model E2 and even more in model E3 (Figures 2e and 2f), where the tidal dissipation rate is the highest, maxima of the surface heat flux are located at the poles and a chain of high heat flux interconnecting the poles is observed. The bottom heat flux is almost zero at the poles and heat out of the core is almost uniquely transported through the equatorial area where a system of cold anomalies is located. The heat extracted from the liquid layer thus strongly varies as a function of latitude. A consequence on the long-term evolution would be that the crystallization of the liquid layer preferentially occurs in the equatorial region.

\subsubsection{Comparison With 1-D Approach}

[51] In order to emphasize the role of lateral variations of effective viscosity in predicting the tidal dissipation, we compare here our calculated strain rates $\dot{\varepsilon}$ with the strain rates $\dot{\varepsilon}_{\text {rad }}$ based on the commonly used approximations considering only a radial dependence of viscosity [e.g., Segatz et al., 1988; Tobie et al., 2005b; Roberts and Nimmo, 2008a]. This comparison clearly indicates that the strain rate and hence the tidal dissipation based on the 1-D viscosity is 
Table 4. Extra Solar Earth Model: Description of Orbital Parameters and Rheological Properties

\begin{tabular}{|c|c|c|c|c|c|c|c|c|c|}
\hline Model & $\begin{array}{c}\eta_{b} \\
(\mathrm{~Pa} \cdot \mathrm{s})\end{array}$ & $a_{\mathrm{vis}}$ & $\begin{array}{c}\hat{\eta}_{b} \\
(\mathrm{~Pa} \cdot \mathrm{s})\end{array}$ & $\hat{a}_{\mathrm{vis}}$ & $e$ & SOPR & $\begin{array}{c}T_{\mathrm{O}} \\
\text { (day) }\end{array}$ & $\begin{array}{c}T_{\mathrm{R}} \\
\text { (day) }\end{array}$ & Q \\
\hline EE1 & $10^{21}$ & 15 & $1.8 \cdot 10^{19}$ & 15 & 0.2 & $3: 2$ & 80 & 53 & 80 \\
\hline EE2 & $10^{21}$ & 15 & $8.8 \cdot 10^{18}$ & 15 & 0.2 & $3: 2$ & 40 & 27 & 80 \\
\hline EE3 & $10^{21}$ & 15 & $9.1 \cdot 10^{18}$ & 15 & 0.2 & $3: 2$ & 30 & 20 & 110 \\
\hline EE4 & $10^{21}$ & 15 & $8.3 \cdot 10^{18}$ & 15 & 0.2 & $3: 2$ & 20 & 13 & 150 \\
\hline EE5 & $10^{21}$ & 15 & $9.1 \cdot 10^{18}$ & 15 & 0.2 & $1: 1$ & 30 & 30 & 110 \\
\hline EE6 & $10^{21}$ & 15 & $2.5 \cdot 10^{17}$ & 15 & 0 & $\mathrm{LR}^{\mathrm{a}}$ & 80 & 1 & 200 \\
\hline
\end{tabular}

${ }^{a}$ LR denotes low spin-orbit period ratio.

underestimated in hot upwellings (Figures $3 \mathrm{~d}-3 \mathrm{f}$ ) and overestimated in cold downwellings (Figures 3j-31) in comparison with the fully $3-\mathrm{D}$ approach.

[52] Previous models of tidally heated convective icy shells [Sotin et al., 2002; Tobie et al., 2003; Showman and Han, 2004; Mitri and Showman, 2008; Roberts and Nimmo, 2008a] have usually adopted a simplified description of tidal dissipation rate computed from the viscosity field, but assuming that the strain rate is uniform or only radially varying. Our calculations show that, in reality, the viscosity significantly modifies the strain rate field. As a consequence, the tidal dissipation rate predicted by the simplified approach is underestimated in hot plumes and overestimated in cold downwellings compared to the 3-D solutions. Our results confirm the analysis of Mitri and Showman [2008] and Han and Showman [2010] who predicted an increase of tidal dissipation rate within hot plumes owing to the enhancement of strain rate induced by the local reductions of viscosity.

[53] As an example, we have computed the dissipated power for model E2, assuming the simplified strain rate based on the 1-D viscosity profile, and the fully 3-D approach. For the same temperature pattern corresponding to $40 \mathrm{My}$ of the time evolution, in hot plumes, the dissipation rate reaches a maximum value of $7.7 \cdot 10^{-7} \mathrm{~W} \cdot \mathrm{m}^{-3}$ for the fully $3-\mathrm{D}$ approach, whereas the maximum value is only $5.3 \cdot 10^{-7} \mathrm{~W} \cdot \mathrm{m}^{-3}$ for the simplified approach. In the cold downwellings and taking into account only the convective part of the mantle (i.e. excluding top $30 \mathrm{~km}$ ), the minimum values of the dissipation rate are $4.1 \cdot 10^{-9} \mathrm{~W}$. $\mathrm{m}^{-3}$ for the 3-D approach and $4.3 \cdot 10^{-8} \mathrm{~W} \cdot \mathrm{m}^{-3}$ for the simplified approach. The difference in the global dissipated power is only $3 \%$ between the two solutions for the same temperature pattern. If a simulation for the model E2 is performed using the simplified approach and starting from the no-heating statistical steady state initial conditions, the global dissipation reaches the value of $4.6 \mathrm{GW}$ for the statistical steady state. The dissipated power is thus underestimated by $\sim 25 \%$ in comparison with the fully $3-D$ approach. Even if not fully correct, the approximation based on local perturbations of tidal dissipation by viscosity is better than spherically symmetric models with no perturbation. However, it leads to a systematic underestimation of tidal dissipation in the hot anomalies, which can significantly affect the long-term evolution of the system.

\subsection{Earth-Like Planets}

[54] Besides the strong dependence of tidal dissipation on rheological parameters shown for Enceladus, other impor- tant parameters determining tidal dissipation also exists. Here, we focus on the influence of the orbital parameters, namely the spin-orbit period ratio (i.e. for a planet locked in $3: 2$ or $1: 1$ resonance or with low spin-orbit period ratio, see Appendix B) and the orbiting period $T_{\mathrm{O}}$. We investigate systematically the conditions under which tidal dissipation becomes a significant source of heat in Earth-like planets. In a first set of simulations, we consider an orbital configuration similar to Mercury ( $e=0.2,3: 2$ SOPR) and vary the orbital period (corresponding to a variation of semi-major axis and/or central star mass in accordance with Kepler's third law). In a second set of numerical experiments, we investigate the effect of different types of orbit, i.e. synchronous eccentric orbit and a body on a circular orbit having low spin-orbit period ratio with a spin period of 1 day. See Table 4 for a summary of model parameters.

[55] The modeled planet is considered to have a composition and characteristics similar to the Earth: silicate mantle, liquid outer core and undeformable inner core (see Table 2). Moreover, we adopt rheological parameters similar to the Earth. The viscosity of the Earth's mantle is, however, still rather uncertain. A wide range of acceptable values of viscosity is usually considered based on inversions of geophysical data [e.g., Peltier, 1996; Lambeck and Johnston, 1998; Ricard et al., 1993; Čadek and Fleitout, 2006] and laboratory experiments [e.g., Karato and Wu, 1993; Hirth and Kohlstedt, 2003; Korenaga and Karato, 2008], the latter pertaining to specific material, the former to global radially averaged profiles. In all our models, we adopt a value of $10^{21} \mathrm{~Pa} \cdot \mathrm{s}$ corresponding to the coremantle boundary (i.e. to the dimensionless temperature 1) and a value of 15 for the viscosity parameter $a_{\text {vis }}$ corresponding to $Q^{*} \approx 500 \mathrm{~kJ} \cdot \mathrm{mol}^{-1}$.

[56] Similarly to the Enceladus case, the initial temperature field comes from the statistical steady state solution for a basally heated spherical shell (temperature at the surface and at the bottom boundary is kept constant) and without any tidal dissipation. Identical initial conditions (in the sense of dimensionless temperature) are considered in all simulations.

[57] For the viscoelastic response, the shear modulus $\mu=$ $200 \mathrm{GPa}$ based on lower mantle values of the model by Dziewonski and Anderson [1981] is used here. The value of the effective viscosity is chosen to predict the observed quality factor $Q$ [Ray et al., 2001; Benjamin et al., 2006]. Moreover, we take into account a slight increase of $Q$ with decreasing excitation period $T_{e}$ as suggested by Benjamin et al. [2006] for the Earth. The minimal effective viscosity (i.e. viscosity at the core-mantle boundary $\hat{\eta}_{b}$ ) is thus defined using equation (4). For simplicity, the effective viscosity parameter $\hat{a}_{\mathrm{vis}}$ is chosen to be equal to $a_{\mathrm{vis}}$. A complete list of all rheological parameters for the viscoelastic response is given in Table 4.

[58] The boundary conditions are similar to the Enceladus case, except for the free-slip condition prescribed on the top boundary for the viscous flow. A resolution corresponding to $128 \times 64 \times 64$ grid points is used in each identical block for solving viscous flow. For the viscoelastic response, the radial resolution is 128 grid points in radial direction and the cut-off degree is $j_{\max }=80$. The convergence parameter defining the relaxed solution is $c=5 \cdot 10^{-8}$. The response to the tidal forcing is updated every 200 MCTS. 
A (EE1)
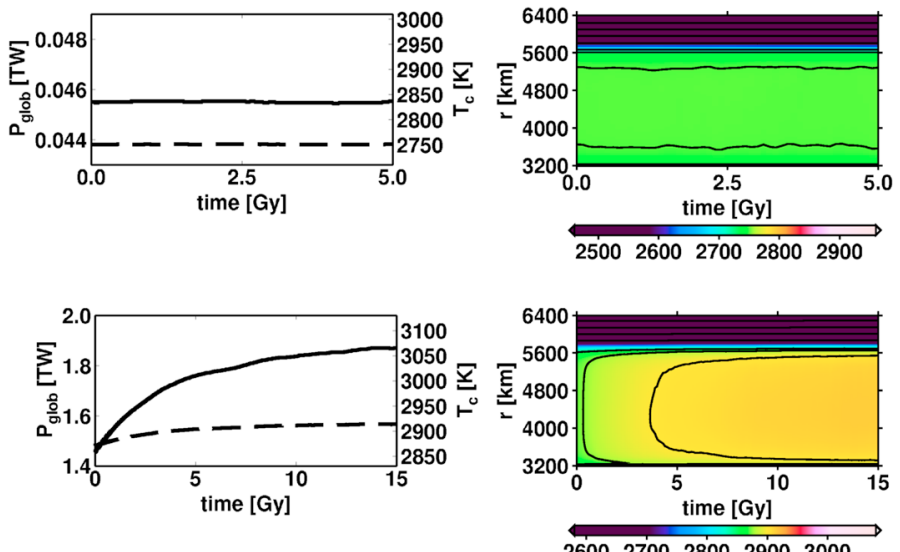

B (EE2)

26002700280029003000
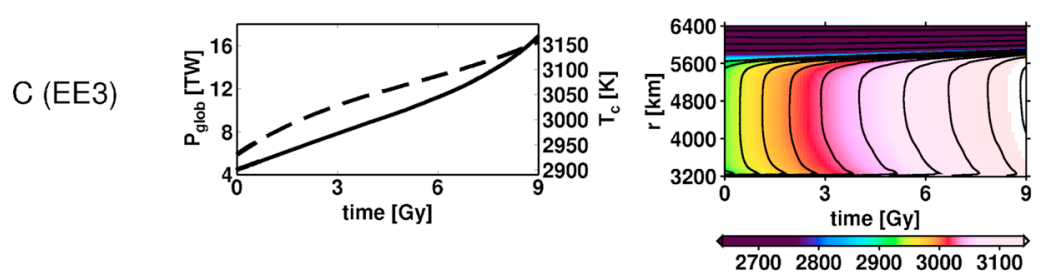

$D(E E 4)$
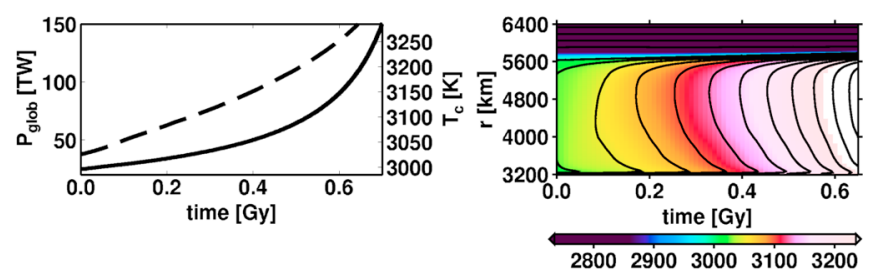

E (EE5)
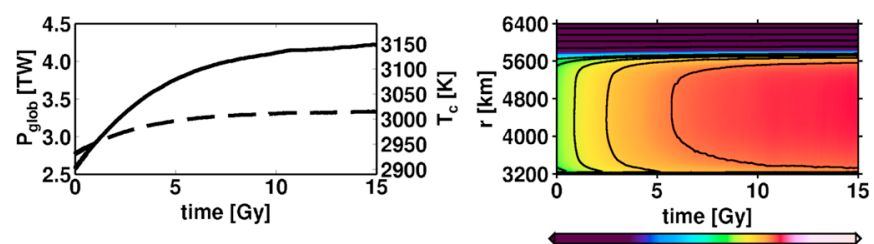

27002800290030003100

$F(E E 6)$
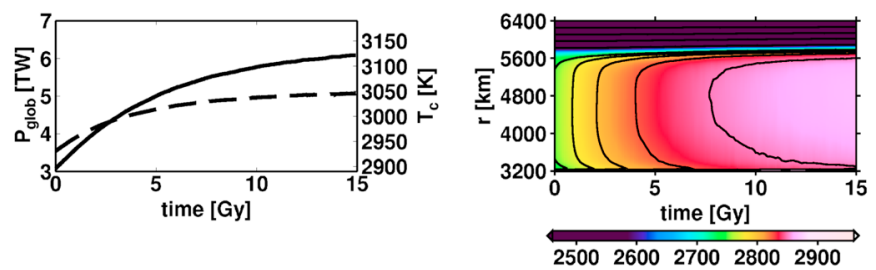

Figure 4. Extra solar Earth model: results for models (a) EE1, (b) EE2, (c) EE3, (d) EE4, (e) EE5, and (f) EE6. (left) Time evolution of the global heating $P_{\text {glob }}$ (solid line) and temperature $T_{\mathrm{c}}$ (dashed line) at middle depth of the shell. (right) Time evolution of the radial temperature profile (in K) with time. Contours are plotted for every $500 \mathrm{~K}$ in the lid and $25 \mathrm{~K}$ in the convective part.

\subsubsection{Global Time Evolution}

[59] Time evolutions for all investigated models are shown in Figure 4. For each model, a different value of surface temperature is considered taking into account the distance from a Sun-like star. For this calculation, a specific assumption has to be considered: the mass of the central body is equal to the mass of the Sun and the same solar constant as for the Sun is used. The surface temperature is then estimated using the Stephan-Boltzmann law with the black body assumption. The surface temperature is then $460 \mathrm{~K}$ for $T_{\mathrm{O}}=80$ days, $580 \mathrm{~K}$ for $T_{\mathrm{O}}=40$ days, $640 \mathrm{~K}$ for $T_{\mathrm{O}}=30$ days and $735 \mathrm{~K}$ for $T_{\mathrm{O}}=20$ days.

[60] For models EE1-EE4 (Figures 4a-4d), dependence of the tidal dissipation and thermal evolution on a value of orbiting period is shown for an orbit locked in 3:2 spin-orbit period ratio and high eccentricity $e=0.2$. For model EE1 
(Figure 4a), the distance between the orbiting planet and its star is characterized by the orbiting period $T_{\mathrm{O}}=80$ days, approximately corresponding to Mercury's orbit. The global tidal dissipation is $\sim 0.045 \mathrm{TW}$ and this value remains almost constant during the whole simulation, i.e. for 5 Gy. Only small perturbations are observed (Figure $4 \mathrm{a}$, left). The effect on the temperature evolution is negligible and no significant changes are induced (Figure 4a, right).

[61] For a planet orbiting closer to the tide generating star $\left(T_{\mathrm{O}}=40\right.$ days, model EE2), the tidal dissipation rate increases by almost two orders of magnitude. The value of the global tidal dissipation is $1.5 \mathrm{TW}$ at the beginning of simulation and increases to a statistical steady state value of 1.9 TW, i.e. by approximately $30 \%$ (see Figure $4 \mathrm{~b}$, left). Similarly to Enceladus, a positive feedback between temperature and global dissipation is observed. The temperature field is in this case slightly influenced by the presence of tidal dissipation and it increases by $\sim 40 \mathrm{~K}$ in the convecting part (see Figure 4b, right). We estimate that this orbital period (or a somewhat lower value), i.e. $T_{\mathrm{O}} \sim 40$ days, corresponds the critical value below which the tidal dissipation has an actual effect on the thermal evolution and internal temperature field in our model set up.

[62] For an even closer body $\left(T_{\mathrm{O}}=30\right.$ days, model EE3), further increase of the tidal dissipation is observed. Initially, the global dissipation is equal to $4.5 \mathrm{TW}$ which is value $\sim 4$ times higher than for model EE2. Due to the positive feedback between temperature and tidal dissipation, this value grows during the simulation and no statistical steady state is reached (Figure 4c, left; the concavity of the curve even suggesting that such an equilibrium will not be obtained in the next billion years). The internal temperature increases gradually and eventually exceeds the temperature at the core-mantle boundary (Figure 4c, right) after $8.5 \mathrm{~Gy}$. Therefore, at the end of the calculations, significant melting is expected almost everywhere in the mantle. The occurrence of melting would significantly reduce the effective viscosity, but at the same time would reduce the effective shear modulus [e.g., Fischer and Spohn, 1990; Moore, 2003]. Estimating the balance of the two competing effect of melt content on tidal dissipation (enhancement due to the viscosity decrease, reduction due to the shear modulus decrease) is difficult in the absence of experimental data at the appropriate frequency range. Melting is also expected to significantly modify heat transfer. Further modeling efforts would be needed to determine the thermal and dissipative state of the mantle when a significant fraction of the temperature field exceeds the solidus. Our approach permits us, however, to determine the conditions under which global scale melting may occur in an Earth-like planet. Even if melting is not considered, our results suggest that an energetic equilibrium can be found only for a relatively narrow interval of orbital period.

[63] For an orbiting period $T_{\mathrm{O}}$ of 20 days (model EE4), the global dissipation is $\sim 25 \mathrm{TW}$ at the beginning and, as expected, it increases during the whole simulation. A steady state is never reached; instead an energy runaway is observed (Figure 4d, left). The temperature grows relatively fast and the internal temperature exceeds the temperature at the core-mantle boundary after $0.6 \mathrm{~Gy}$. Again, a very large amount of melt is expected.
[64] For models EE5 and EE6, different types of tidal potentials are used. For model EE5, the planet is assumed to be locked in 1:1 spin-orbit resonance with an eccentricity of 0.2 and an orbital period of 30 days, equivalent to model EE3. The initial global dissipation is equal to $2.6 \mathrm{TW}$. This value is reduced by almost a factor of two in comparison with the one for model EE3. This slight decrease of global dissipation has, however, an important consequence: an energy equilibrium can be reached for model EE5 and the statistical steady state value at the end of the simulation is 4.2 TW (see Figure 4e, left). The temperature within the convective part of the mantle increases by $\sim 85 \mathrm{~K}$ (see Figure $4 \mathrm{e}$, right).

[65] Here, tidal dissipation is hence larger for a planet locked in 3:2 SOPR (model EE3) than for a planet with synchronous eccentric orbit (model EE5). In general, a planet locked in 3:2 resonance is expected to have a higher dissipation rate than a planet locked in 1:1 resonance for a large range of eccentricities since the leading part of disturbing tidal potential for 3:2 SOPR orbit (see Appendix B) does not depend on eccentricity and, contrary to 1:1 SOPR orbit, the periodic tidal potential is nonzero even for zero eccentricity.

[66] For a model with a low spin-orbit period ratio (LR), the spin period is fixed to 1 day, i.e. to the Earth value. In this model, the spin and orbit periods are independent and this case simulates free rotation. This type of tidal potential is expected to be important during the early stages of evolution of planetary bodies before complete despinning. The characteristic forcing period $T_{e}$ is significantly lower than in the previous cases, specifically $T_{e} \approx 0.5$ day (consult Appendix B). Note that the effective viscosity derived from equation (4) is hence low in comparison with effective viscosities for models EE3 and EE5. For a model with $T_{\mathrm{O}}=$ 30 days, the global dissipation is more than one order of magnitude higher and hence it is not comparable to models having the same orbit period, i.e. models EE3 and EE5. It has been found, that a similar tidal dissipation is reached if a body is much farther from a tide-generating body $\left(T_{\mathrm{O}}=\right.$ 80 days, model EE6). For model EE6, the initial value of global dissipation is $3.1 \mathrm{TW}$. Due to the positive feedback, this value increases to statistical steady state value $6.2 \mathrm{TW}$ (Figure 4f, left). The evolution of temperature profile is shown in (Figure 4f, right), the internal temperature increases by $\sim 115 \mathrm{~K}$ at the end of the simulation.

\subsubsection{Dissipative and Convective Patterns}

[67] Snapshots of temperature field and tidal dissipation for the different models tested (EE2, EE3, EE5 and EE6) are plotted in Figure 5. Only the temperature field just above the core-mantle boundary is shown here (Figure 5a). The corresponding tidal dissipation pattern is displayed in Figure $5 \mathrm{~b}$ and the tidal dissipation pattern in the convecting part of the mantles in Figure 5c. The behavior of the temperature field has characteristics rather similar to the ones of the Enceladus model. At the base of the mantle, hot upwellings tend to concentrate in regions where tidal dissipation is high and cold downwellings are predominantly observed in areas associated with low tidal dissipation (see Figure 5a). These characteristics are, to a lesser extent, observed also in the middle of the mantle where hot anomalies have, however, considerably lower amplitude than cold short-wavelength anomalies. 

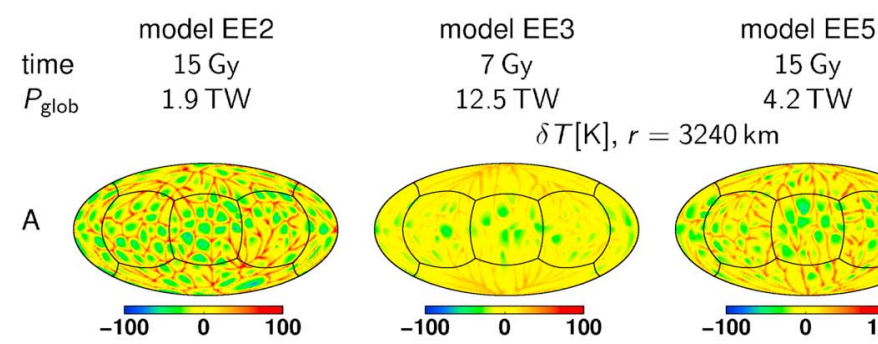

model EE5

15 Gy

4.2 TW
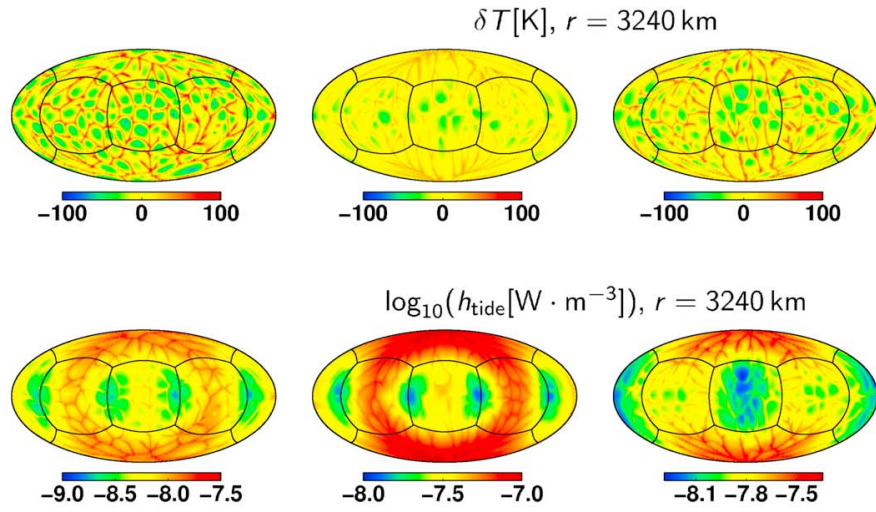

B

C
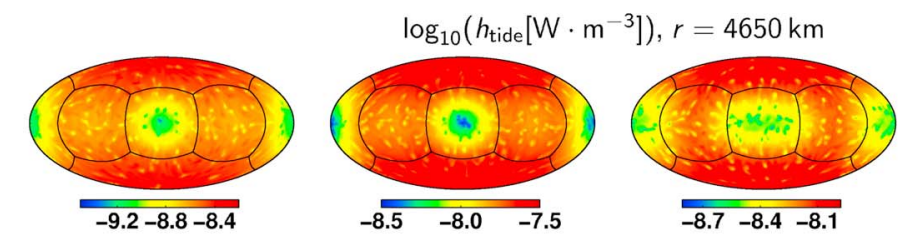

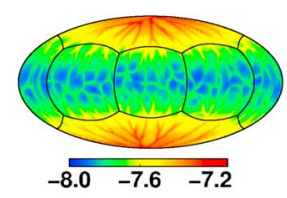

model EE6

15 Gy

$6.1 \mathrm{TW}$
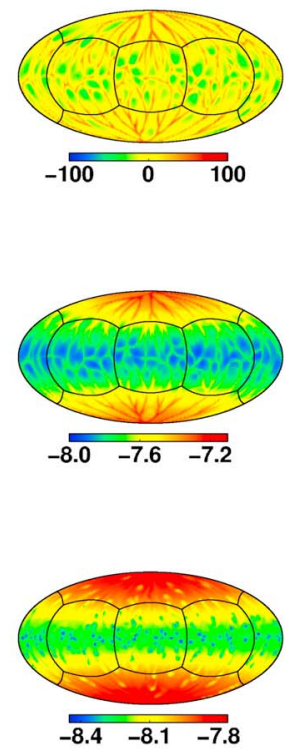

Figure 5. Extra solar Earth model: solution for models EE2, EE3, EE5, and EE6. (a) Temperature anomaly $\delta T$ at the depth $r=3240 \mathrm{~km}$, and $\log 10$ of tidal dissipation at the depth (b) $r=3240 \mathrm{~km}$ and (c) $r=4650 \mathrm{~km}$.

[68] The change of the tidal dissipation pattern among different types of tidal dissipation is demonstrated in Figures $5 \mathrm{~b}$ and $5 \mathrm{c}$. For all types of tidal potential, the characteristics in the latitudinal direction are predominantly determined by boundary conditions, i.e. by decoupling of the solid mantle and the liquid core. Similarly to Enceladus simulations, maxima of tidal dissipation concentrate in the polar regions. For comparison, maxima are located in the equatorial region in the case of solid-solid interface (i.e. for no-slip boundary conditions). In the longitudinal direction, the fluctuations of tidal dissipation is controlled by the type of tidal potential, i.e. by the type of orbit.

[69] For 3:2 SOPR (Figure 5, models EE2 and EE3) and geometrical factor $f=0.5$, the pattern at the base of mantle consists of an annulus of relatively high tidal dissipation connecting both poles. In the equatorial region, two pairs of antipodal minima of tidal dissipation appear. With increasing radius, each pair of minima progressively merge. At midmantle radius (Figure 5c, models EE2 and EE3), only two minima can be observed in the equatorial region whereas in other regions a rather uniform high value with weak maxima at poles is observed. As the temperature is slightly lower in the convecting part of the mantle than at the base of the mantle, the dissipated power is also slightly lower. In the stagnant lid, the dissipation pattern is rather complex and highly perturbed by the temperature anomalies. Its contribution to the overall energy budget is however negligible due to low temperatures and hence high effective viscosity there.

[70] For an eccentric synchronous orbit, two antipodal minima of tidal dissipation are located in the equatorial area at the base of the mantle (Figure 5b, model EE5). In the convecting part at midmantle radius (Figure 5c, model EE5), the minima are still located at the equator but their shape and amplitude changes. For the investigated eccentricity, this pattern is rather similar to the one observed for $3: 2$ SOPR (Figure 5c, models models EE2 and EE3) except for the different shape of the minima on the equator. Hence, for $e \approx 0.2$ and orbital periods which produce approximately the same averaged global dissipation for both 1:1 and 3:2 SOPR orbits, the influence of tidal dissipation on evolution of temperature field will be similar. Note, however, that the patterns are changing with eccentricity especially for body locked in 3:2 SOPR orbit. In this latter case, the longitudinal variations decreases with decreasing eccentricity.

[71] For a model with a low spin-orbit period ratio, contrary to the previous cases, no long wavelength longitudinal fluctuations are observed. The tidal dissipation pattern is almost axisymmetric and it is modulated only by temperature anomalies. Again, at the base of the mantle, the maxima are located at the poles and the minima on the equator (Figure 5b, model EE6). The minimum at the equator is weakening with increasing radius (Figure 5c, model EE6).

\section{Conclusions}

[72] In order to investigate the influence of tidal forcing on thermal evolution of planets and satellites, we have developed a new numerical tool that solves simultaneously the equations for thermal convection and for viscoelastic tidal deformation in a three-dimensional spherical geometry. The first applications of our numerical models to Enceladus and Earth-like planets presented here demonstrate how both orbital characteristics and convective instabilities determine 
the tidal dissipation pattern. Our numerical approach that combines a finite volume method for solving the equation of thermal convection [Choblet et al., 2007] and a discretization based on finite difference in radial direction and spherical harmonics in lateral direction for solving the viscoelastic problem [Tobie et al., 2008] have raised several technical issues. For each issue, we have found a reasonable solution.

[73] A first important technical problem is directly connected to the coupled time steppings: i.e. how often the tidal dissipation should be updated (updating cycle of tidal heating). Since the Courant stability criterion ensuring limited temperature change during one mantle convection step is used, the viscoelastic response is simply updated after a constant number of mantle convection time steps. A second issue is related to the use of the time domain approach for the viscoelastic response. Contrary to the Laplace approach, which is commonly used [Peltier, 1974; Segatz et al., 1988; Tobie et al., 2005b], this allows to include easily 3-D spatial variations of effective viscosity. This, however, brings the question of finding a relaxed solution. Here, we choose a criterion for which the relative change of global dissipation between two individual viscoelastic time steps has to be smaller than a chosen value. The last issue is related to the spatial coupling between the two discrete frameworks used for viscous flow ("cubed sphere" discretization) and the viscoelastic response (discretization using spherical harmonic functions). For the computation of viscoelastic response, a spherical harmonic analysis of the temperature field and a harmonic synthesis for tidal dissipation have to be thus evaluated in each discrete layer for a chosen maximal cut-off degree $j_{\max }$. The coupling between the two different discretizations and methods, nevertheless, brings advantages. The finite volume method is more efficient for solving viscous flow with large lateral viscosity variations. Contrary to viscous flow, the self-gravity cannot be neglected in the viscoelastic deformation. The additional gravity potential caused by the deformation of boundaries can be easily evaluated and used in the spectral approach.

[74] The three main technical parameters, which are the updating cycle of tidal heating, the convergence parameter $c$ and the cut-off degree $j_{\max }$, have been tested in the case of Enceladus. The most crucial parameter is the cut-off degree $j_{\text {max }}$ determining the spatial resolution. We show that a value of $j_{\max }$ approximately corresponding to the spatial resolution of mantle convection has to be employed. The lateral and radial variations of the temperature field inherent to the convection with temperature-dependent rheology influence significantly the distribution of both the deviatoric part of the stress tensor and the tidal heating pattern. In particular, the temperature field is the most sensitive to the chosen value of the cut-off degree in the vicinity of stagnant lid where the largest lateral temperature variations are observed and the tidal dissipation is still high. The frequency at which the tidal dissipation field is reevaluated also affects the evolution of the temperature field, but to a lesser extent. Updating every several hundred time steps appears to be a reasonable updating cycle. The parameter of convergence toward a relaxed solution (equation (19)) has an optimal value of $10^{-7}-10^{-8}$.

[75] Our calculations also show that the long-wavelength characteristics of tidal stress and strain rate are predomi- nantly controlled by the orbital parameters and the presence of a liquid layer. However, the convective planforms strongly modulate the tidal stress and strain rate patterns. By comparing the square of the second invariant of strain rate calculated with our 3-D approach to the one obtained using the classical method based on radially layered interior models [e.g., Segatz et al., 1988; Tobie et al., 2005b; Roberts and Nimmo, 2008a], we show that the tidal strain rate is strongly increased in hot upwellings and decreased in cold downwellings. As a consequence, the classical radially layered technique underestimates tidal strain rate and hence the dissipation in hot upwellings. In the case of Enceladus, we demonstrate that this can result in a difference of 3\% for the same temperature pattern. If the time evolutions are performed, the difference of $25 \%$ in the global energy budget is found between the statistical steady states obtained for the simplified and the fully $3-\mathrm{D}$ cases.

[76] For Enceladus, the global tidally dissipated power is similar to the one inferred from thermal emissions [Spencer et al., 2009] when assuming an effective viscosity of $10^{13} \mathrm{~Pa} \cdot \mathrm{s}$ for the temperature near the melting point. As expected, due to the presence of a liquid layer at the bottom of the ice shell, the tidal heat production is concentrated in the polar regions. This leads to a strong reduction of the heat flux through the ice-ocean interface in the polar region. As a consequence, the crystallization rate of a global ocean is strongly reduced there. This suggests that a liquid reservoir could be sustained in the polar regions. The evolution of such a liquid layer will be addressed in a specific forthcoming study. Based on our simulations, no difference can arise between the north and south poles. Other ingredients (e.g., chemical heterogeneity in the silicate core, anomalous structure inherited from earlier stages) should thus be included to explain the observed strong dichotomy between the two poles. Additional mechanisms including faulting need also to be introduced in order to explain the strong focusing of heat release along the tiger stripes [Roberts and Nimmo, 2008b; Abramov and Spencer, 2009]. These additional mechanisms will be included in the tool presented here in order to provide a coherent description of the observed dynamics of Enceladus' south pole.

[77] For Earth-like planets, we demonstrate the influence of tidal dissipation on thermal evolution for different orbital configurations. Again the presence of a liquid core leads to an enhancement of tidal dissipation toward the poles. The longitudinal variations are entirely determined by the orbital configuration $(1: 1,3: 2$ resonances or free rotation). Note however that zero obliquity is considered in our calculations; nonzero values would modify the tidal dissipation pattern. For an eccentricity of 0.2 , we show that dissipation becomes very large for orbiting periods $<30$ days. In this case, a thermal runaway occurs, thus probably inducing large scale melting of the mantle. The value of the orbiting period below which such thermal runaways may be triggered obviously depends on the orbital configuration (eccentricity, type of resonance) and heat transfer characteristics (Rayleigh number, rheology, melting and possible phase transitions). Moreover, the presence of the radiogenic heating is another important issue which has impact on the global tidal dissipation. Nevertheless, this aspect has been neglected here for the sake of simplicity since the abundance of radiogenic elements in extrasolar systems is uncertain and depends on 
the large variety of physical properties and mechanisms as well as on the history. Note, however, that the presence of significant radiogenic heating leads to an increase of the internal temperature and thus favors the occurrence of thermal runaways. The sensitivity of thermal runaway to orbital and convective characteristics, radiogenic heating as well as to the more complex viscosity including the pressure dependence will be addressed in a forthcoming study. For planetary bodies orbiting at short distance from their stars in $1: 1$ or $3: 2$ resonance, the heterogeneous insolation pattern is likely to affect the near surface thermal structure and therefore could strongly influence both convective and dissipative patterns. Such surface temperature variations should be included in the future to better simulate the tidal dissipation pattern of such bodies. The new method presented here will provide a valuable tool to assess whether tidally triggered large scale magmatism occurs on Earth-like planets which will be detected around neighboring stars in the near future.

\section{Appendix A: Nondimensional Form of the Governing Equations}

[78] For a better understanding and readability of the equations and parameters, the scaling into nondimensional variables is usually considered especially in the case of viscous flow. The system (6-8) may be thus rewritten into

$$
\begin{gathered}
0=\nabla^{\prime} \cdot \boldsymbol{v}^{\prime}, \\
0=-\nabla^{\prime} p^{\prime}+\nabla^{\prime} \cdot\left(\eta^{\prime}\left(\theta^{\prime}\right)\left(\nabla^{\prime} \boldsymbol{v}^{\prime}+\nabla^{\prime \mathrm{T}} \boldsymbol{v}^{\prime}\right)\right)-R a \theta^{\prime} \boldsymbol{e}_{r}, \\
\frac{\partial \theta^{\prime}}{\partial t^{\prime}}=-\boldsymbol{v}^{\prime} \cdot \nabla^{\prime} \theta^{\prime}+\nabla^{\prime 2} \theta^{\prime}+h_{i}^{\prime}+h_{\text {tide }}^{\prime}
\end{gathered}
$$

where prime indicates dimensionless variables (see Table 1) and operators. Since we consider here homogeneous boundary conditions (no-slip or free-slip), the boundary conditions for equations (A1) and (A2) have the same form as the dimensional ones. The boundary conditions for temperature may be rewritten into $\theta\left(r^{\prime}=r_{t}^{\prime}=1, t^{\prime}\right)=0$ and $\theta\left(r^{\prime}=\right.$ $\left.r_{b}^{\prime}=\frac{f}{1-f}, t^{\prime}\right)=1$. The vigor of the system is characterized by Rayleigh number $R a$ and viscosity parameter $a_{\text {vis }}$ together with the heating $h_{i}^{\prime}$ and $h_{\text {tide }}^{\prime}$.

[79] The viscoelastic response (equations (9)-(11)) determining the budget of tidal dissipation $h_{\text {tide }}$ may be nondimensionalized as follows:

$$
\begin{gathered}
0=\nabla^{\prime} \cdot \boldsymbol{u}^{\prime}, \\
0=-\nabla^{\prime} \pi^{\prime}+\nabla^{\prime} \cdot \mathbf{D}^{\prime}+\boldsymbol{f}^{\prime}, \\
-\frac{\hat{\mu}^{\prime}}{\hat{\eta}^{\prime}} \mathbf{D}^{\prime}=\frac{\partial \mathbf{D}^{\prime}}{\partial \tau^{\prime}}-\frac{\partial}{\partial \tau^{\prime}}\left[\hat{\mu}^{\prime}\left(\nabla^{\prime} \boldsymbol{u}^{\prime}+\nabla^{\prime \mathrm{T}} \boldsymbol{u}^{\prime}\right)\right] .
\end{gathered}
$$

The nondimensional variables are scaled by the effective gravitational rigidity $\rho_{0} g_{0} d$ (see Table 1 ). The scaling is more complex than in the case of viscous flow due to the nature of the body force which is specific to each individual planet (radius of body and thickness of mantle, eccentricity, rotational and orbit period), self-gravitation and due to the nonhomogeneous boundary conditions.
[80] The dimensionless scaling of the body force and boundary conditions may be rearranged into:

body force:

$$
\boldsymbol{f}^{\prime}=\frac{1}{g_{0}}(\nabla \Theta+\nabla V)=\frac{d}{\tau_{0}^{2} g_{0}}\left(\nabla^{\prime} \Theta^{\prime}+\nabla^{\prime} V^{\prime}\right),
$$

surface boundary conditions:

$$
\left(-\pi^{\prime} \mathbf{I}+\mathbf{D}^{\prime}\right) \cdot \boldsymbol{e}_{r}-u_{r}^{\prime} \frac{g_{\mathrm{S}}}{g_{0}} \boldsymbol{e}_{r}=\mathbf{0}
$$

boundary conditions for solid-liquid interface:

$$
\begin{gathered}
\left(-\pi^{\prime} \mathbf{I}+\mathbf{D}^{\prime}\right) \cdot \boldsymbol{e}_{r}-u_{r}{ }^{\prime} \frac{\rho_{\text {core }}-\rho_{0}}{\rho_{0}} \frac{g_{\mathrm{B}}}{g_{0}} \boldsymbol{e}_{r} \\
=\frac{\rho_{\text {core }}}{\rho_{0}} \frac{d}{\tau_{0}^{2} g_{0}}\left(\Theta^{\prime}+V^{\prime}\right) \boldsymbol{e}_{r},
\end{gathered}
$$

bottom boundary conditions (solid-solid):

$$
\boldsymbol{u}^{\prime}=\mathbf{0} .
$$

\section{Appendix B: Periodic Part of Tidal Potentials}

[81] The following formulas for periodic parts of tidal potentials are derived from Kaula [1964] for the general case and for different spin-orbit period ratios (SOPR 1:1 and $3: 2$ ) up to the fourth order in the eccentricity $e ; n^{*}$ denotes the mean motion, $\omega$ is the angular (spin) velocity of a body, $\tau$ the time and $P_{20}$ and $P_{22}$ denote the fully normalized associated Legendre functions.

\section{general SOPR}

$$
\begin{aligned}
-\frac{1}{2}\left(n^{*}\right)^{2} r^{2}\left\{P _ { 2 0 } ( \operatorname { c o s } \vartheta ) \left[3 e \cos \left(n^{*} \tau\right)+\frac{9}{2} e^{2} \cos \left(2 n^{*} \tau\right)\right.\right. \\
\left.+\frac{27}{8} e^{3} \cos \left(n^{*} \tau\right)+\frac{7}{2} e^{4} \cos \left(2 n^{*} \tau\right)\right] \\
\quad-\frac{1}{2} P_{22}(\cos \vartheta)\left[\left(-\frac{1}{2} e+\frac{1}{16} e^{3}\right) \cos \left(2 \varphi+2 \omega \tau-n^{*} \tau\right)\right. \\
+\left(1-\frac{5}{2} e^{2}+\frac{13}{16} e^{4}\right) \cos \left(2 \varphi+2 \omega \tau-2 n^{*} \tau\right) \\
+\left(\frac{7}{2} e-\frac{123}{16} e^{3}\right) \cos \left(2 \varphi+2 \omega \tau-3 n^{*} \tau\right) \\
\left.\left.\quad+\left(\frac{17}{2} e^{2}-\frac{115}{16} e^{4}\right) \cos \left(2 \varphi+2 \omega \tau-4 n^{*} \tau\right)\right]\right\}
\end{aligned}
$$

1:1 SOPR

$$
\begin{aligned}
- & \frac{1}{2}\left(n^{*}\right)^{2} r^{2}\left\{P _ { 2 0 } ( \operatorname { c o s } \vartheta ) \left[3 e \cos \left(n^{*} \tau\right)+\frac{9}{2} e^{2} \cos \left(2 n^{*} \tau\right)\right.\right. \\
& \left.+\frac{27}{8} e^{3} \cos \left(n^{*} \tau\right)+\frac{7}{2} e^{4} \cos \left(2 n^{*} \tau\right)\right] \\
& -\frac{1}{2} P_{22}(\cos \vartheta)\left[e\left(3 \cos (2 \varphi) \cos \left(n^{*} \tau\right)+4 \sin (2 \varphi) \sin \left(n^{*} \tau\right)\right)\right. \\
& +\frac{17}{2} e^{2}\left(\cos (2 \varphi) \cos \left(2 n^{*} \tau\right)+\sin (2 \varphi) \sin \left(2 n^{*} \tau\right)\right) \\
& -\frac{1}{8} e^{3}\left(61 \cos (2 \varphi) \cos \left(n^{*} \tau\right)+62 \sin (2 \varphi) \sin \left(n^{*} \tau\right)\right) \\
& \left.\left.-\frac{115}{6} e^{4}\left(\cos (2 \varphi) \cos \left(2 n^{*} \tau\right)+\sin (2 \varphi) \sin \left(2 n^{*} \tau\right)\right)\right]\right\}
\end{aligned}
$$


a)

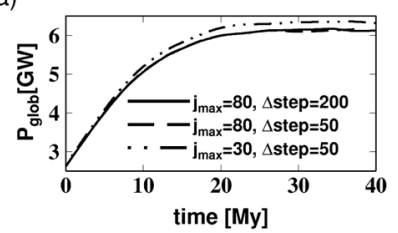

c)

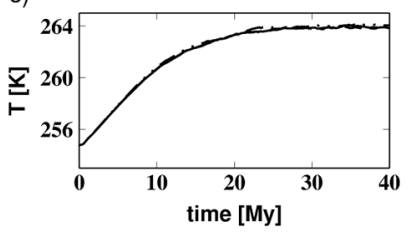

b)

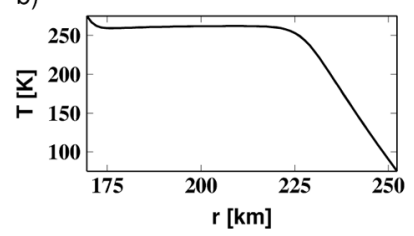

d)

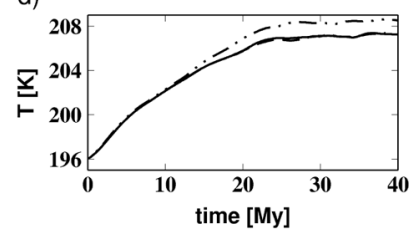

Figure C1. Enceladus: sensitivity tests for model E2. (a) Time dependence of global average dissipation $P_{\text {glob}}$, (b) temperature profile at 40 My (all three lines overlap), (c-d) time dependence of the average temperature at the depths $r=172 \mathrm{~km}$ and $r=235 \mathrm{~km}$; solid line denotes reference model (E2) with cut-off degree $j_{\max }=80$ and viscoelastic response updated every $\Delta$ step $=200$ MCTS, dashed line denotes $j_{\max }=80$ and $\Delta$ step $=50$ MCTS, dash-dotted line denotes $j_{\max }=30$ and $\Delta$ step $=50$ MCTS.

\section{$3: 2$ SOPR}

$$
\begin{aligned}
-\frac{1}{2}\left(n^{*}\right. & )^{2} r^{2}\left\{P _ { 2 0 } ( \operatorname { c o s } \vartheta ) \left[3 e \cos \left(n^{*} \tau\right)+\frac{9}{2} e^{2} \cos \left(2 n^{*} \tau\right)\right.\right. \\
& \left.+\frac{27}{8} e^{3} \cos \left(n^{*} \tau\right)+\frac{7}{2} e^{4} \cos \left(2 n^{*} \tau\right)\right] \\
& -\frac{1}{2} P_{22}(\cos \vartheta)\left[\left(\cos (2 \varphi) \cos \left(n^{*} \tau\right)-\sin (2 \varphi) \sin \left(n^{*} \tau\right)\right)\right. \\
& -\frac{1}{2} e\left(\cos (2 \varphi) \cos \left(2 n^{*} \tau\right)-\sin (2 \varphi) \sin \left(2 n^{*} \tau\right)\right) \\
& +e^{2}\left(6 \cos (2 \varphi) \cos \left(n^{*} \tau\right)+11 \sin (2 \varphi) \sin \left(n^{*} \tau\right)\right) \\
& +\frac{1}{16} e^{3}\left(\cos (2 \varphi) \cos \left(2 n^{*} \tau\right)-\sin (2 \varphi) \sin \left(2 n^{*} \tau\right)\right) \\
& -\frac{1}{48} e^{4}\left(881 \cos (2 \varphi) \cos \left(n^{*} \tau\right)\right. \\
& \left.\left.\left.+959 \sin (2 \varphi) \sin \left(2 n^{*} \tau\right)\right)\right]\right\} .
\end{aligned}
$$

\section{Appendix C: Benchmark and Sensitivity Tests}

\section{C1. Benchmark}

[82] The numerical implementations of both methods described above have been carefully tested. The benchmark and performed tests (including performance efficiency) for the mantle convection part are described by Choblet [2005] and Choblet et al. [2007].

[83] The original implementation of viscoelastic problem [Tobie et al., 2008] has been tested for the case of surface loading against finite element code ZEBULON (http://www. nwnumerics.com/Zebulon) and against the code by Martinec [2000] using Lagrangian formulation of equations (9)-(11) and spectral-finite element discretization. Moreover, the evaluation of product $\mathbf{D} / \hat{\eta}$ using the Legendre-Fourier trans-

form has been compared to the computation using the analytical formula [Jones, 1985]. For the tidal forcing, series of tests have been performed against the code described by Tobie et al. [2005b] based on technique classically used to describe spheroidal oscillations for layered interior models [e.g., Takeushi and Saito, 1972]. This method solves the viscoelastic response to tides together with the LaplacePoisson equation for gravitational potential in the whole sphere and allows radially stratified models to be studied. Additionally, the efficiency of the convergence criterion in order to find the relaxed solution may be compared to this method using Laplace domain since this approach naturally gives a solution for $\tau \rightarrow \infty$. Special attention has been paid to the examples presented here. For the bodies described in section 4 , we have found that the period average of the components of displacement as well as the tidal dissipation in the mantle differ from the solution of Tobie et al. [2005b] by less than $3 \%$.

\section{C2. Sensitivity Tests for Numerical Parameters}

[84] A series of tests have been performed to determine the sensitivity of the solution to the newly introduced numerical parameters, i.e. the convergence factor $c$, the updating cycle of the tidal deformation and the cut-off degree $j_{\max }$. Specifically, several simulations have been run for a model of Enceladus with significant tidal heating (model E2, see Tables 2 and 3) and the resulting characteristics of simulations for different numerical parameters have been compared.

[85] In the case of the convergence factor $c$, two simulations with 3-D rheological properties (one with reference numerical parameters $c=5 \cdot 10^{-7}$, and the second one with a more strict convergence parameter, $c=5 \cdot 10^{-9}$ ) have been run in order to analyze the sensitivity to this factor. Only negligible influence of this parameter on the results has been observed. The differences are less than $0.01 \%$ for both the global dissipation and the average temperature in each layer during the whole simulation (i.e. until the statistical steady solution is found).

[86] Similar tests have been carried out for the frequency of updating the tidal dissipation (i.e. updating the viscoelastic response). Two values $200 \mathrm{MCTS}$ and $50 \mathrm{MCTS}$ have been tested. If the identical temperature field is considered at the beginning then the difference between these two solutions is $\sim 0.05 \%$ after 200 MCTS for both the global heating and the temperature profile (i.e. the heating for the reference model is somewhat smaller). Since the temperature pattern starts to slightly differ with increasing number of MCTS these differences cumulate and increase with time. During the simulations, the difference for global dissipation oscillates between $-0.8 \%$ and $0.8 \%$ reflecting a chaotic behavior of the system. Similar oscillations are also observed for the temperature profile, where the maximum difference is $0.4 \%$ (oscillates between $-0.4 \%$ and $0.2 \%$ ). This maximum difference is found in the vicinity of the stagnant lid while the maximum difference is $\sim 0.2 \%$ in the rest of the mantle. The influence of the frequency of updating the tidal dissipation on the global dissipation and temperature is shown in Figure C1.

[87] The lateral resolution of the viscoelastic response model (i.e. the value of cut-off degree $j_{\max }$ ) has been found to be a parameter which the results are the most sensitive to. For an analysis of this effect, we have performed the sim- 
ulation for model E2 using $j_{\max }=80$ (reference model) and $j_{\max }=30$. For the lower resolution, the heating is overestimated (by a factor of two) within plumes and underestimated (by 30\%) in their neighborhood if the same temperature field is considered. During the whole simulations, the difference for global dissipation oscillates between $2 \%$ and $5 \%$ (the heat production is systematically higher than in the reference model). The differences between temperature profiles again depend on radius. The maximum difference $(2 \%)$ is found in the vicinity of the stagnant lid whereas differences of $\sim 0.2 \%$ are observed in the rest of the mantle. Again, the influence of $j_{\max }$ on the global dissipation and temperature is illustrated in Figure C1.

[88] These sensitivity tests indicate that the updating cycle of 200 MCTS is probably sufficient and a smaller value of 50 MCTS is more time consuming and would not significantly improve the solution. Concerning the spatial discretization, it appears that the cut-off value $j_{\max }$ of 80 should be employed in this case in order to correctly describe the dissipation in short-wavelengths hot plumes where most of the dissipation occurs. In general, the choice of an appropriate value of $j_{\max }$ depends on the rheological properties of the system and it increases with increasing Rayleigh number Ra.

[89] Acknowledgments. We thank Lijie Han, Francis Nimmo, and James Roberts for their comments and suggestions that helped to improve the manuscript. This work was supported by the Czech Science Foundation project P210/10/P306, the Research Program MSM0021620860 of the Ministryof Education of the Czech Republic, ETHER project of the Agence Nationale de la Recherche (ANR, France), and INSU's Programme National de Planétologie (France). The computations were carried out using CCIPL and CINES computational facilities. A part of the work was done during a post-doc of M.B. in LPG Nantes.

\section{References}

Abramov, O., and J. R. Spencer (2009), Endogenic heat from Enceladus' south polar fractures: New observations, and models of conductive surface heating, Icarus, 199, 189-196, doi:10.1016/j.icarus.2008.07.016.

Ashby, M. F., and R. A. Verrall (1978), Micromechanism of flow and fracture, and their relevance to the rheology of the upper mantle, Philos. Trans. R. Soc. London A., 288, 59-95, doi:10.1098/rsta.1978.0006.

Barr, A. C. (2008), Mobile lid convection beneath Enceladus' south polar terrain, J. Geophys. Res., 113, E07009, doi:10.1029/2008JE003114.

Benjamin, D., J. Wahr, R. D. Ray, G. D. Egbert, and S. D. Desai (2006), Constraints on mantle anelasticity from geodetic observations and implications for the $\mathrm{J} 2$ anomaly, Geophys. J. Int., 165, 3-16, doi:10.1111/ j.1365-246X.2006.02915.x.

Budd, W. F., and T. H. Jacka (1989), A review of ice rheology for ice-sheet modeling, Cold Reg. Sci. Technol., 16, 107-144, doi:10.1016/0165-232X (89)90014-1.

Cadek, O. (1989), Spherical harmonic approach to the solution of the mantle stress problem, Stud. Geophys. Geod., 33, 177-197, doi:10.1007/ BF01646583.

Čadek, O. (2003), Viscoelastic relaxation of spherical Earth with a 3-D viscosity structure, paper presented at EGS-AGU-EUG Joint Assembly, p. 5279, Nice, France.

Čadek, O., and L. Fleitout (2006), Effects of lateral viscosity variations in $\mathrm{D}^{\prime \prime}$ on predictions of the long-wavelength geoid, Stud. Geophys. Geod., 50, 217-232, doi:10.1007/s11200-006-0013-0.

Čadek, O., Z. Martinec, and C. Matyska (1992), Spectral variational approach to the non-Newtonian Stokes problem in a spherical shell, Computer. Phys. Commun., 71, 56-70, doi:10.1016/0010-4655(92)90072-7.

Choblet, G. (2005), Modelling thermal convection with large viscosity gradients in one block of the 'cubed sphere,' J. Comput. Phys., 205, 269-291, doi:10.1016/j.jcp.2004.11.005.

Choblet, G., and C. Sotin (2000), 3-D thermal convection with variable viscosity: Can transient cooling be described by a quasi-static scaling law?, Phys. Earth Planet. Inter., 119, 321-336, doi:10.1016/S0031-9201(00) 00136-9.
Choblet, G., O. Čadek, F. Couturier, and C. Dumoulin (2007), (EDIPUS: A new tool to study the dynamics of planetary interiors, Geophys. J. Int., 170, 9-30, doi:10.1111/j.1365-246X.2007.03419.x.

Cole, D. M. (1995), A model for the anelastic straining of saline ice subjected to cyclic loading, Phys. Mag. A, 72, 231-248, doi:10.1080/ 01418619508239592 .

Czechowski, L., and J. Leliwa-Kopystynski (2005), Convection driven by tidal and radiogenic heating in medium size icy satellites, Planet. Space Sci., 53, 749-769, doi:10.1016/j.pss.2005.01.004.

Deschamps, F., and C. Sotin (2001), Thermal convection in the outer shell of large icy satellites, J. Geophys. Res., 106, 5107-5121, doi:10.1029/ 2000JE001253.

Durham, W. B., and L. A. Stern (2001), Rheological properties of water ice: Applications to satellites of the outer planets, Annu. Rev. Earth Planet Sci., 29, 295-330, doi:10.1146/annurev.earth.29.1.295.

Dziewonski, A. M., and D. L. Anderson (1981), Preliminary reference Earth model, Phys. Earth Planet. Inter., 24, 297-356, doi:10.1016/ 0031-9201(81)90046-7.

Faul, U. H., and I. Jackson (2005), The seismological signature of temperature and grain size variations in the upper mantle, Earth Planet. Sci. Lett., 234, 119-134, doi:10.1016/j.epsl.2005.02.008.

Fischer, H.-J., and T. Spohn (1990), Thermal-orbital histories of viscoelastic models of Io (J1), Icarus, 83, 39-65, doi:10.1016/0019-1035(90) 90005-T.

Goldsby, D. L., and D. L. Kohlstedt (2001), Superplastic deformation of ice: Experimental observations, J. Geophys. Res., 106, 11,017-11,030, doi:10.1029/2000JB900336.

Han, L., and A. Showman (2010), Coupled convection and tidal dissipation in Europa's ice shell, Icarus, 207, 834-844, doi:10.1016/j. icarus.2009.12.028.

Hanyk, L., C. Matyska, and D. Yuen (2005), Short time-scale heating on the Earth's mantle by ice-sheet dynamics, Earth Planets Space, 57, 895-902.

Hirth, G., and D. Kohlstedt (2003), Rheology of the upper mantle and the mantle wedge: A view from the experimentalists, in Inside the Subduction Factory, Geophys. Monogr. Ser., vol. 138, edited by J. Eiler, pp. 85-105, AGU, Washington, D. C.

Hussmann, H., and T. Spohn (2004), Thermal-orbital evolution of Io and Europa, Icarus, 171, 391-410, doi:10.1016/j.icarus.2004.05.020.

Hussmann, H., T. Spohn, and K. Wieczerkowski (2002), Thermal equilibrium states of Europas ice shell: Implications for internal ocean thickness and surface heat flow, Icarus, 156, 143-151, doi:10.1006/icar.2001.6776.

Jackson, B., R. Greenberg, and R. Barnes (2008), Tidal heating of extrasolar planets, Astrophys. J., 681, 1631-1638, doi:10.1086/587641.

Jones, M. (1985), Spherical Harmonics and Tensors for Classical Field Theory, 230 pp., Res. Stud. Press, Letchworth, U. K.

Karato, S.-I., and P. Wu (1993), Rheology of the upper mantle: A synthesis, Science, 260, 83-108, doi:10.1126/science.260.5109.771.

Kaula, W. (1964), Tidal dissipation by solid friction and the resulting orbital evolution, Rev. Geophys., 2, 661-685, doi:10.1029/ RG002i004p00661.

Korenaga, J., and S.-I. Karato (2008), A new analysis of experimental data on olivine rheology, J. Geophys. Res., 113, B02403, doi:10.1029/ 2007JB005100.

Lainey, V., J. Arlot, Ö. Karatekin, and T. van Hoolst (2009), Strong tidal dissipation in Io and Jupiter from astrometric observations, Nature, 459, 957-959, doi:10.1038/nature08108.

Lambeck, K., and P. Johnston (1998), The viscosity of the mantle: evidence from analysis of glacial-rebound phenomena, in The Earth's Mantle: Composition, Structure and Evolution, edited by I. Jackson, pp. 461502, Cambridge Univ. Press, New York.

Lopes, R. M. C., and D. A. Williams (2005), Io after Galileo, Rep. Prog. Phys., 68, 303-340, doi:10.1088/0034-4885/68/2/R02.

Mardling, R. A., and D. Lin (2004), On the survival of short-period terrestrial planets, Astrophys. J., 614, 955-959, doi:10.1086/423794.

Martinec, Z. (1989), Program to calculate the spectral harmonic expansion coefficients of the two scalar fields product, Comput. Phys. Commun., 54, 177-182, doi:10.1016/0010-4655(89)90043-X.

Martinec, Z. (2000), Spectral-finite element approach to three-dimensional viscoelastic relaxation in a spherical Earth, Geophys. J. Int., 142, $117-$ 141, doi:10.1046/j.1365-246x.2000.00138.x.

Mitri, G., and A. P. Showman (2008), A model for the temperaturedependence of tidal dissipation in convective plumes on icy satellites: Implications for Europa and Enceladus, Icarus, 196, 758-764, doi:10.1016/j.icarus.2008.01.010.

Moore, W. (2003), Tidal heating and convection in Io, J. Geophys. Res., 108(E8), 5096, doi:10.1029/2002JE001943.

Ojakangas, W., and D. Stevenson (1989), Thermal state of an ice shell on Europa, Icarus, 81, 220-241, doi:10.1016/0019-1035(89)90052-3. 
Parmentier, E., C. Sotin, and B. Travis (1994), Turbulent 3-D thermal convection in an infinite Prandtl number, volumetrically heated fluid: Implications for mantle dynamics, Geophys. J. Int., 116, 241-251, doi:10.1111/j.1365-246X.1994.tb01795.x

Peale, S., P. Cassen, and R. Reynolds (1979), Melting of Io by tidal dissipation, Science, 203, 892-894, doi:10.1126/science.203.4383.892.

Peltier, W. (1974), Impulse response of a Maxwell Earth, Rev. Geophys., 12, 649-669, doi:10.1029/RG012i004p00649.

Peltier, W. (1996), Mantle viscosity and ice-age ice sheet tomography, Science, 273, 1359-1364, doi:10.1126/science.273.5280.1359.

Porco, C., et al. (2006), Cassini observes the active south pole of Enceladus, Science, 311, 1393-1401, doi:10.1126/science.273.5280.1359.

Press, W., S. Teukolsky, W. Vetterling, and B. Flannery (1992), Numerical Recipes in FORTRAN: The Art of Scientific Computing, 2nd ed. Cambridge Univ. Press, New York.

Ray, R. D., R. J. Eanes, and F. G. Lemoine (2001), Constraints on energy dissipation in the Earth's body tide from satellite tracking and altimetry, Geophys. J. Int., 144, 471-480, doi:10.1046/j.1365-246x.2001.00356.x.

Reese, C. C., V. S. Solomatov, and L.-N. Moresi (1999), Non-Newtonian stagnant lid convection and magmatic resurfacing on Venus, Icarus, 139 , 67-80, doi:10.1006/icar.1999.6088.

Ricard, Y., M. Richards, C. Lithgow-Bertelloni, and Y. Le Stunff (1993), A geodynamic model of mantle density heterogeneity, J. Geophys. Res., 98 , 21,895-21,909, doi:10.1029/93JB02216.

Roberts, J., and F. Nimmo (2008a), Tidal heating and the long-term stability of a subsurface ocean on Enceladus, Icarus, 194, 675-689, doi:10.1016/j.icarus.2007.11.010.

Roberts, J., and F. Nimmo (2008b), Near-surface heating on Enceladus and the south polar thermal anomaly, Geophys. Res. Lett., 35, L09201, doi:10.1029/2008GL033725.

Robuchon, G., G. Tobie, G. Choblet, O. Čadek, and A. Mocquet (2009), Thermal evolution of Mercury: Implication for despinning and contraction, Lunar Planet. Sci., XL, Abstract 1866.

Ronchi, C., R. Iacono, and P. S. Paolucci (1996), The "cubed sphere": A new method for the solution of partial differential equations in spherical geometry, J. Comput. Phys., 124(1), 93-114, doi:10.1006/jcph.1996.0047.

Ross, M. N., and G. Schubert (1989), Evolution of the lunar orbit with temperature- and frequency-dependent dissipation, J. Geophys. Res., 94, 9533-9544, doi:10.1029/JB094iB07p09533.

Schubert, G., D. Turcotte, and P. Olson (2001), Mantle Convection in the Earth and Planets, Cambridge Univ. Press, New York.

Segatz, M., T. Spohn, M. N. Ross, and G. Schubert (1988), Tidal dissipation, surface heat flow, and figure of viscoelastic models of Io, Icarus, 75, 187-206, doi:10.1016/0019-1035(88)90001-2.

Showman, A. P., and L. Han (2004), Numerical simulations of convection in Europa's ice shell: Implications for surface features, J. Geophys. Res., 109, E01010, doi:10.1029/2003JE002103.

Sotin, C., J. Head, and G. Tobie (2002), Europa: Tidal heating of upwelling thermal plumes and the origin of lenticulae and chaos melting, Geophys. Res. Lett., 29(8), 1233, doi:10.1029/2001GL013844.

Sotin, C., G. Tobie, J. Wahr, and W. B. McKinnon (2009), Tides and tidal heating on Europa, in Europa After Galileo, edited by R. T. Pappalardo et al., pp. 85-117, Univ. of Ariz. Press, Tucson.
Spencer, J., C. Howett, J. Pearl, and M. Segura (2009), High-resolution observations of Enceladus' thermal radiation in 2008, Eos Trans. $A G U, 90(22)$, Jt. Assem. Suppl., P33A-04.

Tackley, P. (2008), Modelling compressible mantle convection with large viscosity contrasts in a three-dimensional spherical shell using the yinyang grid, Phys. Earth Planet. Inter., 171, 7-18, doi:10.1016/j. pepi.2008.08.005

Tackley, P., G. Schubert, G. Glatzmaier, P. Schenk, J. Ratcliff, and J.-P. Matas (2001), Three-dimensional simulations of mantle convection in Io, Icarus, 149, 79-93, doi:10.1006/icar.2000.6536.

Takeushi, H., and M. Saito (1972), Seismic surfaces waves, in Seismology: Surface Waves and Earth Oscillations, Methods Comput. Phys., vol. 11, edited by B. A. Bolt et al., pp. 217-295, Academic, New York.

Tatibouet, J., J. Perez, and R. Vassoille (1987), Study of grain-boundaries in ice by internal friction measurements, J. Phys., 48, 197-203.

Tobie, G., G. Choblet, and C. Sotin (2003), Tidally heated convection: Constraints on Europa's ice shell thickness, J. Geophys. Res., 108 (E11), 5124, doi:10.1029/2003JE002099.

Tobie, G., O. Grasset, J. Lunine, A. Mocquet, and C. Sotin (2005a), Titan's internal structure inferred from a coupled thermal-orbital model, Icarus, 175, 496-502, doi:10.1016/j.icarus.2004.12.007.

Tobie, G., A. Mocquet, and C. Sotin (2005b), Tidal dissipation within large icy satellites: Applications to Europa and Titan, Icarus, 177, 534-549, doi:10.1016/j.icarus.2005.04.006.

Tobie, G., J. I. Lunine, and C. Sotin (2006), Episodic outgassing as the origin of atmospheric methane on Titan, Nature, 440, 61-64, doi:10.1038/ nature04497.

Tobie, G., O. Čadek, and C. Sotin (2008), Solid tidal friction above a liquid water reservoir as the origin of the south pole hotspot on Enceladus, Icarus, 196, 642-652, doi:10.1016/j.icarus.2008.03.008

Varshalovich, D., A. Moskalev, and V. Khersonskii (1989), Quantum Theory of Angular Momentum, World Sci., Singapore.

Veeder, G., D. Matson, T. Johnson, D. Blaney, and J. Goguen (1994), Io's heat flow from infrared radiometry: 1983-1993, J. Geophys. Res., 99 , 17,095-17,162, doi:10.1029/94JE00637.

Yoder, C., A. Konopliv, D. Yuan, E. Standish, and W. Folkner (2003), Fluid core size of Mars from detection of the solar tide, Science, 300 299-303, doi:10.1126/science.1079645.

Zhong, S., A. McNamara, E. Tan, L. Moresi, and M. Gurnis (2008), A benchmark study on mantle convection in a 3-D spherical shell using CitcomS Geochem. Geophys. Geosyst., 9, Q10017, doi:10.1029/2008GC002048.

M. Běhounková and O. Čadek, Faculty of Mathematics and Physics, Department of Geophysics, Charles University in Prague, V Holešovičkách 2, 18000 Praha 8, Czech Republic. (behounek@karel.troja. mff.cuni.cz; oc@karel.troja.mff.cuni.cz)

G. Choblet and G. Tobie, Laboratoire de Planétologie et Géodynamique, UMR 6112, Université de Nantes, CNRS, 2 rue de la Houssinière, BP 92208, F-44322 Nantes CEDEX 3, France. (gael.choblet@univ-nantes.fr; gabriel.tobie@univ-nantes.fr) 